\title{
Derivation of Hamiltonians for Accelerators
}

by Keith R. Symon

September 1997
RECEVED

DEC $0 \backslash 1997$

OS TI 
Argonne National Laboratory, with facilities in the states of Illinois and Idaho, is owned by the United States government, and operated by The University of Chicago under the provisions of a contract with the Department of Energy.

\section{DISCLAIMER}

This report was prepared as an account of work sponsored by an agency of the United States Government. Neither the United States Government nor any agency thereof, nor any of their employees, makes any warranty, express or implied, or assumes any legal liability or responsibility for the accuracy, completeness, or usefulness of any information, apparatus, product, or process disclosed, or represents that its use would not infringe privately owned rights. Reference herein to any specific commercial product, process, or service by trade name, trademark, manufacturer, or otherwise, does not necessarily constitute or imply its endorsement, recommendation, or favoring by the United States Government or any agency thereof. The views and opinions of authors expressed herein do not necessarily state or reflect those of the United States Government or any agency thereof.

Reproduced from the best available copy.

Available to DOE and DOE contractors from the

Office of Scientific and Technical Information

$$
\text { P.O. Box } 62
$$

Oak Ridge, TN 37831

Prices available from (423) 576-8401

Available to the public from the

National Technical Information Service

U.S. Department of Commerce

5285 Port Royal Road

Springfield, VA 22161 
Distribution Category: Atomic, Molecular, and Chemical Physics (UC-411)

\section{ARGONNE NATIONAL LABORATORY \\ 9700 South Cass Avenue \\ Argonne, Illinois 60439}

ANL/APS/TB-28

\section{DERIVATION OF HAMILTONIANS FOR ACCELERATORS}

Keith R. Symon ${ }^{1}$

Argonne National Laboratory

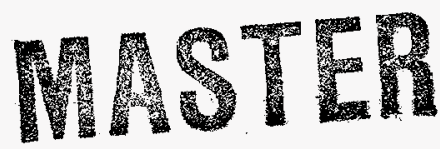

DETREUTION OF THS DOCUNENT IS UHEMTED

September 12, 1997

work sponsored by

\section{U.S. DEPARTMENT OF ENERGY \\ Office of Energy Research}

${ }^{1}$ Permanent address: University of Wisconsin-Madison 


\section{DESCLAmisx}

Portions of this document migy be illegibie in electronic imige produets. Imsges are produced from the best availabie origion doemenent 


\section{Contents}

1 INTRODUCTION 1

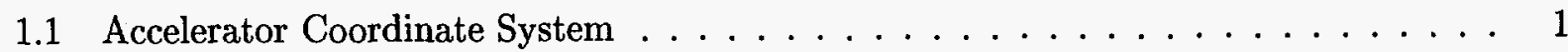

1.2 Handy Formulas . . . . . . . . . . . . . . . . . . . . . . . 1

1.3 The Complete Hamiltonians . . . . . . . . . . . . . . . . . 2

2 THE ELECTROMAGNETIC POTENTIALS FOR LATTICE ELEMENTS 3

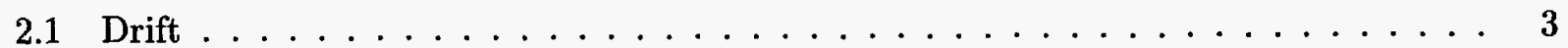

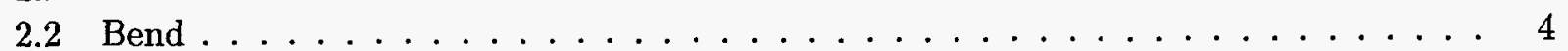

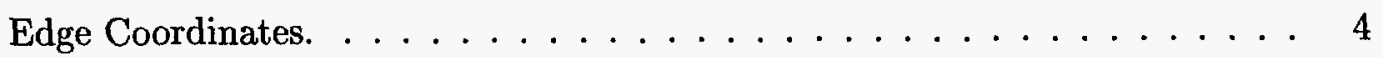

Translation from Edge to Accelerator Coordinates. . . . . . . . . . . 5

The Term $\hat{\mathbf{y}} B(z) \ldots \ldots \ldots \ldots \ldots \ldots \ldots \ldots$

The Remaining Terms. . . . . . . . . . . . . . . . 6

Canonical Potential for a Bend. . . . . . . . . . . . . . 7

Thin Edge Perpendicular to Orbit. . . . . . . . . . . . . . . 7

2.3 Bend with Built-in Gradient . . . . . . . . . . . . . . . . 7

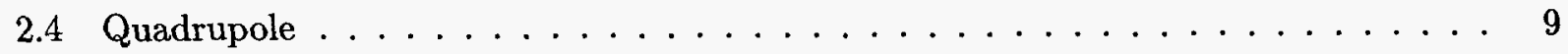

2.5 Skew Quadrupole . . . . . . . . . . . . . . . . . . . 10

Check of Quadrupole Formulas. . . . . . . . . . . . . . . . . . 11

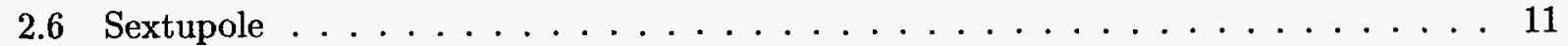

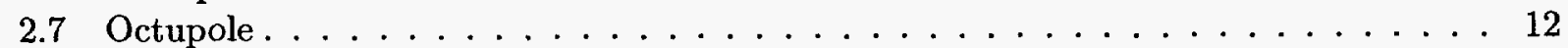

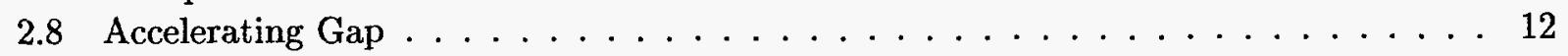

2.9 Space-Charge Potentials . . . . . . . . . . . . . . . . 15

3 TRANSVERSE MOTION $\quad 17$

3.1 Space-Charge Terms . . . . . . . . . . . . . . . . . . . 17

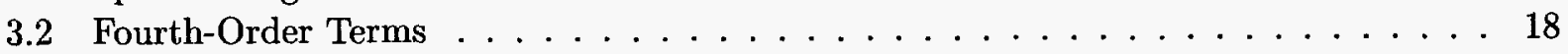

4 LONGITUDINAL MOTION $\quad 19$

4.1 Second-Order Hamiltonian with Constant Radio Frequency . . . . . . . . . . 19

Synchrotron Variables. . . . . . . . . . . . . . . . 19

The Hamiltonian. . . . . . . . . . . . . . . . . . . . . . 20

Difference Equations of Motion. . . . . . . . . . . . . . . . 21

Smoothed Hamiltonian. . . . . . . . . . . . . . . . 22

4.2 Exact Canonical Variables for Time Varying Radio Frequency . . . . . . . . . . . . 22

5 SYNCHROBETATRON COUPLING $\quad 24$

5.1 Coupling Hamiltonian in Original Variables . . . . . . . . . . . . . . . . 24

5.2 Transformation to Synchronous Coordinates . . . . . . . . . . . . 25

5.3 Transformation to Betatron Coordinates . . . . . . . . . . . . 30

$\begin{array}{ll}\text { REFERENCES } & \mathbf{3 4}\end{array}$ 


\section{INTRODUCTION}

In this report various forms of the Hamiltonian for particle motion in an accelerator will be derived. Many of these forms will be used as a section in a forthcoming Handbook of Accelerator Physics and Engineering, edited by Alex Chao and Maury Tigner. Derivations of many of these formulas can be found elsewhere, for example in studies of nonlinear dynamics $[1,2,3,4,5,6,7]$.

Except where noted, the treatment will apply generally to linear and circular accelerators, storage rings, and beamlines. The generic term accelerator will be used to refer to any of these devices. We will use the usual accelerator coordinate system [1], which will be introduced first, along with a list of handy formulas. We then start from the general Hamiltonian for a particle in an electromagnetic field, using the accelerator coordinate system, with time $t$ as independent variable. We switch to a form more convenient for most purposes using the distance $s$ along the reference orbit as independent variable.

In section 2 , formulas will be derived for the vector potentials that describe the various lattice components. In sections 3,4 , and 5 , special forms of the Hamiltonian will be derived for transverse horizontal and vertical motion, for longitudinal motion, and for synchrobetatron coupling of horizontal and longitudinal motions. Hamiltonians will be expanded to fourth order in the variables.

\subsection{Accelerator Coordinate System}

We will use the standard accelerator coordinate system shown in Fig. 1.1. It is based on a specified reference orbit. The coordinates are:

$x$ - the distance measured horizontally outward from the reference orbit,

$y$ - the distance measured vertically, and

$s$ - the distance measured along the reference orbit from some reference point.

The reference orbit lies in the $x, s$ plane. It is traced out by a particle with suitable initial conditions in the ideal accelerator lattice as designed. The kinetic momentum $\beta \gamma m c$ on the reference orbit will be denoted by $p_{0}$. Magnet errors, misalignments, etc., which can be introduced later may make the reference orbit no longer an actual particle orbit. In a linear accelerator or straight beamline, the reference orbit is a straight line down the center of the focusing magnets and accelerating gaps. There are no transverse fields on the reference orbit. The reference momentum $p_{0}(s)$ is a function of position along the orbit; for a beamline it is constant. In a circular accelerator or storage ring or a beamline with bends, the reference orbit will consist of arcs and straight sections. In a circular accelerator or storage ring, the reference orbit is closed but not necessarily a circle. The reference momentum $p_{0}(t)$ is a function of time or may be constant. The radius $\rho$ is the local radius of curvature of the reference orbit. It is infinite except in a bend. In a bend, we require

$$
p_{0}=e B \rho,
$$

where $B$ is the magnetic field at the reference orbit and must be vertical.

\subsection{Handy Formulas}

We will make use of some handy formulas in accelerator coordinates:

$$
\frac{d \hat{\mathbf{x}}}{d s}=\frac{\hat{\mathbf{s}}}{\rho}, \frac{d \hat{\mathbf{y}}}{d s}=0, \frac{d \hat{\mathbf{s}}}{d s}=-\frac{\hat{\mathbf{x}}}{\rho}, d \mathbf{r}=\hat{\mathbf{x}} d x+\hat{\mathbf{y}} d y+\hat{\mathbf{s}}\left(1+\rho^{-1} x\right) d s,
$$




$$
\begin{aligned}
\nabla= & \hat{\mathbf{x}} \frac{\partial}{\partial x}+\hat{\mathbf{y}} \frac{\partial}{\partial y}+\frac{\hat{\mathbf{s}}}{\left(1+\rho^{-1} x\right)} \frac{\partial}{\partial s} \\
\nabla^{2}= & \frac{1}{\left(1+\rho^{-1} x\right)} \frac{\partial}{\partial x}\left(1+\rho^{-1} x\right) \frac{\partial}{\partial x}+\frac{\partial^{2}}{\partial y^{2}}+\frac{1}{\left(1+\rho^{-1} x\right)} \frac{\partial}{\partial s} \frac{1}{\left(1+\rho^{-1} x\right)} \frac{\partial}{\partial s} \\
\nabla \cdot \mathbf{B}= & \frac{1}{\left(1+\rho^{-1} x\right)} \frac{\partial}{\partial x}\left(1+\rho^{-1} x\right) B_{x}+\frac{\partial B_{y}}{\partial y}+\frac{1}{\left(1+\rho^{-1} x\right)} \frac{\partial B_{s}}{\partial s} \\
\nabla \times \mathbf{A}= & \hat{\mathbf{x}}\left(\frac{\partial A_{s}}{\partial y}-\frac{1}{\left(1+\rho^{-1} x\right)} \frac{\partial A_{y}}{\partial s}\right)+\hat{\mathbf{y}}\left(\frac{1}{\left(1+\rho^{-1} x\right)} \frac{\partial A_{x}}{\partial s}-\frac{1}{\left(1+\rho^{-1} x\right)} \frac{\partial}{\partial x}\left(1+\rho^{-1} x\right) A_{s}\right) \\
& +\hat{\mathbf{s}}\left(\frac{\partial A_{y}}{\partial x}-\frac{\partial A_{x}}{\partial y}\right)
\end{aligned}
$$

where $\hat{\mathbf{x}}, \hat{\mathbf{y}}, \hat{\mathbf{s}}$ are unit vectors in the direction of increase of the corresponding coordinates.

\subsection{The Complete Hamiltonians [1, 2]}

The Hamiltonian for a particle of charge $e$, mass $m$, moving in an electromagnetic field described by electromagnetic potentials $\mathbf{A}_{\mathrm{em}}, \phi$ is

$$
H_{t}\left(x, p_{x}, y, p_{y}, s, p_{s} ; t\right)=e \phi+T,
$$

where

$$
\begin{aligned}
T & =c\left[m^{2} c^{2}+\left(p_{x}-e A_{x}\right)^{2}+\left(p_{y}-e A_{y}\right)^{2}+\left(\frac{p_{s}-e A_{s}}{1+\rho^{-1} x}\right)^{2}\right]^{1 / 2} \\
& =\gamma m c^{2}
\end{aligned}
$$

is the kinetic energy (including rest energy) of the particle. The canonical vector potentials are defined by

$$
\begin{aligned}
A_{x} & =\hat{\mathbf{x}} \cdot \mathbf{A}_{\mathrm{em}} \\
A_{y} & =\hat{\mathbf{y}} \cdot \mathbf{A}_{\mathrm{em}} \\
A_{s} & =\left(1+\rho^{-1} x\right) \hat{\mathbf{s}} \cdot \mathbf{A}_{\mathrm{em}} .
\end{aligned}
$$

For a circular accelerator, all quantities are periodic functions of $s$.

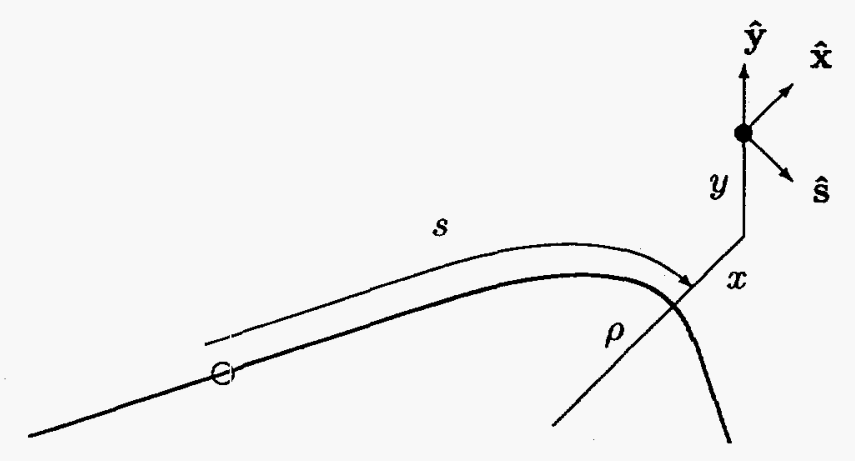

Figure 1.1: Coordinates Relative to Reference Orbit. 
With $s$ as the independent variable, the Hamiltonian is obtained by solving Eq. (1.7) for $p_{s}$ with $H_{t}$ set equal to $E$. The Hamiltonian is the negative of the momentum canonically conjugate to the independent variable:

$$
H_{s}\left(x, p_{x}, y, p_{y}, t,-E ; s\right)=-p_{s}=-e A_{s}-\left(1+\rho^{-1} x\right) P_{s}
$$

where $-E$ is the momentum conjugate to the coordinate $t$ and

$$
\begin{aligned}
P_{s} & =\left[\left(\frac{E-e \phi}{c}\right)^{2}-\left(p_{x}-e A_{x}\right)^{2}-\left(p_{y}-e A_{y}\right)^{2}-m^{2} c^{2}\right]^{1 / 2} \\
& =\beta_{s} \gamma m c
\end{aligned}
$$

is the kinetic momentum in the $s$ direction.

\section{THE ELECTROMAGNETIC POTENTIALS FOR LATTICE ELEMENTS}

We next work out the canonical potentials for magnetic lattice elements, expanded to fourth order in $x, y$. We will choose a gauge in which $A_{y}=0$ and will keep as few terms as possible in $A_{x}$. Other choices of gauge are possible.

The following derivations include the magnet edge effects. Edge corrections depend on the details of the edge design. In each case, a typical edge design has been assumed, as explained in each of the following subsections. For other edge designs, the formulas derived below should give approximate values of the coefficients, particularly if the edges are thin, but they do not include any terms that violate the assumed symmetries.

We will specify the position, length, and edge shape for a bend, quad, sextupole, etc., by giving the field or its gradient as a function of $s$ along the reference orbit. In the subsections below for each case, we derive from this function the magnetic field in the magnet gap and from that the potential to be used in the Hamiltonian.

As the mathematically sophisticated reader will recognize, because the magnetic potential $\psi$ satisfies Laplace's equation, the appropriate (Dirichlet) boundary condition is to specify $\psi$ on the magnet surface or some other closed surface surrounding the region of interest [8]. However this requires specifying the details of the magnet design and probably requires a numerical solution of Laplace's equation. To avoid this and to get approximate analytic formulas for the desired potentials, we have chosen to specify the field in the neighborhood of the reference orbit (essentially a Cauchy condition) and to develop the solution from there in a power series in $x, y$. Such a series will not in general converge unless the field we start with in the neighborhood of the reference orbit is part of the exact solution of Laplace's equation for some magnet design. However, we expect that if the field we start with is a reasonable approximation to the true field along the reference orbit, then the first few terms of the series should be a reasonable approximation to the true field. A similar remark applies to all the derivations in this section.

\subsection{Drift}

For a drift, there is no magnetic field:

$$
\mathbf{A}=0
$$




\subsection{Bend}

We consider a bend with a uniform vertical field $B$ in the interior. We assume a straight edge designed so that there is no field parallel to the edge and no dependence of the fields on the coordinate parallel to the edge. If the field is produced by magnetic pole pieces, the pole face near the edge is traced out by a moving straight line that remains horizontal and parallel to the edge. We will further assume that the magnet is more than twice as long as the edge thickness, so that the edge fields from the two ends do not overlap. We may then consider each edge separately.

Edge Coordinates. Near an edge, we choose a rectangular coordinate system $X, y, z$, with $z$ measured perpendicular to the magnet edge and $X$ parallel to the edge as in Fig. 2.1, which also shows the reference orbit and the relations between accelerator coordinates $s, x$ and the coordinates $X, z$. On the median plane, the field is vertical and depends only on the coordinate $z$ :

$$
\mathbf{B}=\hat{\mathbf{y}} B(z) .
$$

The function $B(z)$ specifies the form of the edge field.

Because there is no magnet current in the magnet gap, we have $\nabla \times \mathbf{B}=0$, and we can derive B from a scalar potential $\psi$ :

$$
\mathbf{B}=\nabla \psi=\nabla \times \mathbf{A}_{\mathrm{em}}
$$

We need the vector potential to fourth order, so we need $\psi$ to fourth order and $\mathbf{B}$ to third order. Because of median plane symmetry, only odd terms in $y$ appear in the expansion of $\psi$ :

$$
\psi(y, z)=B(z) y+\psi_{3}(z) y^{3}+\cdots
$$

Because $\nabla \cdot \mathbf{B}=0$

$$
\nabla^{2} \psi=B_{z z} y+6 \psi_{3} y+\cdots=0
$$

from which we get

$$
\begin{aligned}
\psi_{3} & =-\frac{1}{6} B_{z z} \\
\psi & =B y-\frac{1}{6} B_{z z} y^{3}+\cdots,
\end{aligned}
$$

where subscripts $z$ denote derivatives.

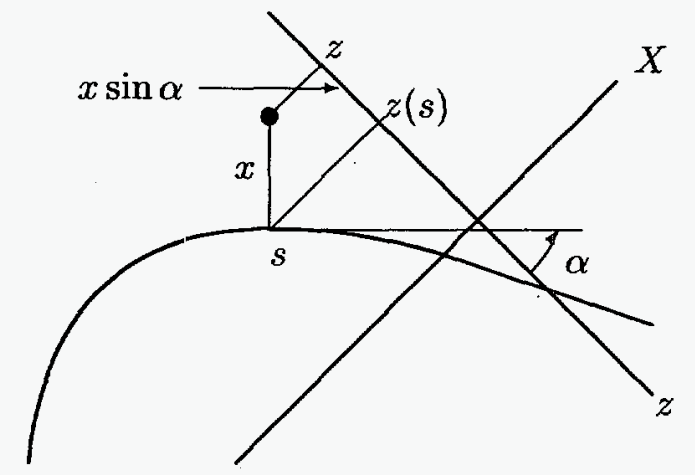

Figure 2.1: Edge Coordinates $X, z$ in the Median Plane. 
Equation (2.7) gives the magnetic field

$$
\mathbf{B}=\hat{\mathbf{y}}\left[B-\frac{1}{2} B_{z z} y^{2}+\cdots\right]+\hat{\mathbf{z}}\left[B_{z} y-\frac{1}{6} B_{z z z} y^{3}+\cdots\right]
$$

We choose a gauge in which the vector potential has only an $X$ component:

$$
\mathbf{B}=\hat{\mathbf{y}} \frac{\partial A_{X}}{\partial z}-\hat{\mathbf{z}} \frac{\partial A_{X}}{\partial y} .
$$

This gives

$$
\mathbf{A}_{\mathrm{em}}=\hat{\mathbf{X}}\left[\int^{z} B\left(z^{\prime}\right) d z^{\prime}-\frac{1}{2} B_{z} y^{2}+\frac{1}{24} B_{z z z} y^{4}+\cdots\right]
$$

Translation from Edge to Accelerator Coordinates. We now have to translate A into accelerator coordinates. Figure 2.1 shows the relation between the accelerator coordinates and the edge coordinates. Fortunately the $y$ coordinate (not shown) is the same in both coordinate systems. A particle is shown at the point $(s, x)$ in accelerator coordinates, $(z, X)$ in edge coordinates. We define the coordinate $z(s)$ of the point $s$ on the reference orbit and the angle $\alpha(s)$ between the tangent to the reference orbit at $s$ and the normal to the edge (i.e., the $z$ axis). We see from Fig. 2.1 that

$$
z=z(s)-x \sin \alpha(s)
$$

We may now expand

$$
\begin{aligned}
B(z) & =B(z(s)-x \sin \alpha) \\
& =B-B_{z} x \sin \alpha+\frac{1}{2} B_{z z} x^{2} \sin ^{2} \alpha-\frac{1}{6} B_{z z z} x^{3} \sin ^{3} \alpha+\cdots
\end{aligned}
$$

where $B$ 's without an argument are evaluated at the point $s$ on the reference orbit. Along the reference orbit, we can see from Fig. 2.1 that

$$
\begin{aligned}
\frac{d z}{d s} & =\cos \alpha, \\
\frac{d \alpha}{d s} & =-\frac{1}{\rho}, \\
\frac{d \rho}{d s} & =-\frac{\rho}{B} \frac{d B}{d s},
\end{aligned}
$$

where the last equation follows from $B \rho=$ constant. Note that Eq. (2.14) can be used to determine $\alpha(s)$ if $\alpha$ is known at some point, say at the magnet edge. For thin edges, $\alpha$ can usually be treated as constant. We can now calculate derivatives along the reference orbit:

$$
\begin{aligned}
& B^{\prime}=\frac{d B}{d s}=B_{z} \frac{d z}{d s}=B_{z} \cos \alpha \\
& B_{1}=B_{z}=\frac{B^{\prime}}{\cos \alpha}, \\
& B^{\prime \prime}=\frac{d B^{\prime}}{d s}=B_{z z} \cos ^{2} \alpha+\frac{B_{z} \sin \alpha}{\rho}=B_{z z} \cos ^{2} \alpha+\frac{B^{\prime} \sin \alpha}{\rho \cos \alpha}, \\
& B_{2}=B_{z z}=\frac{B^{\prime \prime}}{\cos ^{2} \alpha}-\frac{B^{\prime} \sin \alpha}{\rho \cos ^{3} \alpha},
\end{aligned}
$$




$$
\begin{aligned}
B^{\prime \prime \prime} & =\frac{d B^{\prime \prime}}{d s}=B_{z z z} \cos ^{3} \alpha+\frac{2 B_{z z} \cos \alpha \sin \alpha}{\rho}+\left(\frac{B^{\prime} \tan \alpha}{\rho}\right)^{\prime} \\
& =B_{z z z} \cos ^{3} \alpha+\frac{3 B^{\prime \prime} \sin \alpha}{\rho \cos \alpha}-\frac{2 B^{\prime} \sin ^{2} \alpha}{\rho^{2} \cos ^{2} \alpha}-\frac{B^{\prime}}{\rho^{2} \cos ^{2} \alpha}+\frac{B^{\prime 2} \tan \alpha}{B \rho}, \\
B_{3} & =B_{z z z}=\frac{B^{\prime \prime \prime}}{\cos ^{3} \alpha}-\frac{3 B^{\prime \prime} \sin \alpha}{\rho \cos ^{4} \alpha}+\frac{B^{\prime}\left(1+2 \sin ^{2} \alpha\right)}{\rho^{2} \cos ^{5} \alpha}-\frac{B^{2} \sin \alpha}{B \rho \cos ^{4} \alpha}
\end{aligned}
$$

where primes denote derivatives with respect to $s$ and appear in edge corrections.

The Term $\hat{\mathbf{y}} B(z)$. The first term on the right in Eq. (2.10) is awkward to handle in its given form. We note that it gives the field (2.2):

$$
\nabla \times \mathbf{A}_{\mathrm{em} 1}=\nabla \times \hat{\mathbf{X}} \int^{z} B\left(z^{\prime}\right) d z^{\prime}=\hat{\mathbf{y}} B(z)
$$

We therefore seek a formula in accelerator coordinates that gives the field $\hat{\mathbf{y}} B(z)$. According to formula (1.6), if we choose a gauge in which the new $\mathbf{A}_{1}$ has only an $s$ component, we must solve the equation

$$
\frac{1}{1+\rho^{-1} x} \frac{\partial}{\partial x}\left(1+\rho^{-1} x\right) A_{\mathrm{em} 1 s}=\frac{1}{1+\rho^{-1} x} \frac{\partial}{\partial x} A_{1 s}=-B(z)
$$

where $B(z)$ is given by Eq. (2.12). The solution is

$$
A_{1 s}=-B x+\left(\frac{B_{1} \sin \alpha}{2}-\frac{B}{2 \rho}\right) x^{2}+\left(\frac{B_{1} \sin \alpha}{3 \rho}-\frac{B_{2} \sin ^{2} \alpha}{6}\right) x^{3}+\left(\frac{B_{3} \sin ^{3} \alpha}{24}-\frac{B_{2} \sin ^{2} \alpha}{8 \rho}\right) x^{4}
$$

The Remaining Terms. The remainder of $\mathbf{A}_{\mathrm{em}}$ from Eq. (2.10) is, always keeping terms up to fourth order,

$$
\mathbf{A}_{\mathrm{em} 2}=\hat{\mathbf{X}}\left[-\frac{1}{2} B_{z}(z) y^{2}+\frac{1}{24} B_{z z z}(z) y^{4}\right]
$$

From Fig. 2.1,

$$
\hat{\mathbf{X}}=\hat{\mathbf{x}} \cos \alpha+\hat{\mathbf{s}} \sin \alpha .
$$

The coefficients in Eq. (2.25) are to be evaluated at the position $z$ of the particle, so we need to expand them as we did $B(z)$ [Eq. (2.12)], keeping terms up to the appropriate order:

$$
\begin{aligned}
B_{z}(z) & =B_{z}-B_{z z} x \sin \alpha+\frac{1}{2} B_{z z z} x^{2} \sin ^{2} \alpha \\
& =B_{1}-B_{2} x \sin \alpha+\frac{1}{2} B_{3} x^{2} \sin ^{2} \alpha \\
B_{z z z}(z) & =B_{z z z} \\
& =B_{3} .
\end{aligned}
$$

We can now write out $\mathbf{A}_{2}$, using Eqis. (2.25)-(2.28):

$$
\mathbf{A}_{2}=\left(\hat{\mathbf{x}} \cos \alpha+\left(1+\rho^{-1} x\right) \hat{\mathbf{s}} \sin \alpha\right)\left(-\frac{B_{1}}{2} y^{2}+\frac{B_{2} \sin \alpha}{2} x y^{2}-\frac{B_{3} \sin ^{2} \alpha}{4} x^{2} y^{2}+\frac{B_{3}}{24} y^{4}\right) \text {. }
$$


Canonical Potential for a Bend. We collect $\mathbf{A}=\mathbf{A}_{1}+\mathbf{A}_{2}$ to get the canonical magnetic potential for a bend:

$$
\begin{aligned}
\mathbf{A}= & \hat{\mathbf{s}}\left[-B x+\left(\frac{B_{1} \sin \alpha}{2}-\frac{B}{2 \rho}\right) x^{2}-\frac{B_{1} \sin \alpha}{2} y^{2}\right. \\
& +\left(\frac{B_{1} \sin \alpha}{3 \rho}-\frac{B_{2} \sin ^{2} \alpha}{6}\right) x^{3}+\left(\frac{B_{2} \sin ^{2} \alpha}{2}-\frac{B_{1} \sin \alpha}{2 \rho}\right) x y^{2} \\
& \left.+\left(\frac{B_{3} \sin ^{3} \alpha}{24}-\frac{B_{2} \sin ^{2} \alpha}{8 \rho}\right) x^{4}+\left(\frac{B_{2} \sin ^{2} \alpha}{2 \rho}-\frac{B_{3} \sin ^{3} \alpha}{4}\right) x^{2} y^{2}+\frac{B_{3} \sin \alpha}{24} y^{4}\right] \\
& +\hat{\mathbf{x}}\left[-\frac{B_{1} \cos \alpha}{2} y^{2}+\frac{B_{2} \sin \alpha \cos \alpha}{2} x y^{2}-\frac{B_{3} \sin ^{2} \alpha \cos \alpha}{4} x^{2} y^{2}+\frac{B_{3} \cos \alpha}{24} y^{4}\right] .
\end{aligned}
$$

The above formulas for the edge effects have been derived for the right end of the magnet. The reader may verify that we get the same formulas if we consider the left end of the magnet. Note that, at both ends, the positive $z$ axis is in the direction of increasing $s$ and that the angle $\alpha(s)$ is measured counterclockwise from the positive $z$ axis to the tangent to the reference orbit at $s$.

Thin Edge Perpendicular to Orbit. Formula (2.30) is vastly simplified in the case of a thin edge perpendicular to the reference orbit. In that case, we may put $\alpha=0$ (neglecting the variation of $\alpha$ along the orbit in the edge region):

$$
\mathbf{A}=\hat{\mathbf{s}}\left[-B x-\frac{B}{2 \rho} x^{2}\right]+\hat{\mathbf{x}}\left[-\frac{B^{\prime}}{2} y^{2}+\left(\frac{B^{\prime \prime \prime}}{24}+\frac{B^{\prime}}{24 \rho^{2}}\right) y^{4}\right]
$$

\subsection{Bend with Built-in Gradient}

A bending magnet that includes a built-in gradient cannot be treated as in the preceding subsection, because the field is not uniform in the interior of the magnet. We will assume an edge that is perpendicular to the reference orbit. Otherwise the treatment becomes quite difficult and depends sensitively on exactly what assumptions we make about the edge. Such magnets are usually built along the arc of a circle, but we will assume it is built along the reference orbit, so that we may use accelerator coordinates from the beginning. The difference is negligible if the edges are not too thick.

We assume the magnet pole faces are so shaped that in the median plane the field gradient depends only on $s$ :

$$
\mathbf{B}=\hat{\mathbf{y}}[B(s)+G(s) x] .
$$

We will derive separately the two parts of the field.

To get the field

$$
\mathbf{B}_{B 0}=\hat{\mathbf{y}} B(s)
$$

we need the potential

$$
\psi_{B 1}=B(s) y
$$

Formula (1.4) gives

$$
\nabla^{2} \psi_{B 1}=B^{\prime \prime} y+\cdots
$$

so we add the next term:

$$
\psi_{B 3}=B(s) y-\frac{B^{\prime \prime} y^{3}}{6}
$$


This gives

$$
\nabla^{2} \psi_{E 3}=-\left(\frac{2 B^{\prime \prime}}{\rho}+\frac{B^{\prime 2}}{B \rho}\right) x y+\cdots
$$

so we add the next term:

$$
\psi_{B 4}=B(3) y-\frac{B^{\prime \prime} y^{3}}{6}+\frac{1}{6}\left(\frac{2 B^{\prime \prime}}{\rho}+\frac{B^{\prime 2}}{B \rho}\right) x y^{3} .
$$

Fourth order is as far as we need to go. The field is, if we use Eq. (1.3),

$$
\begin{aligned}
\mathbf{B}_{B}= & \nabla \psi_{B 4} \\
= & \hat{\mathbf{y}}\left[B-\frac{B^{\prime \prime} y^{2}}{2}+\left(\frac{B^{\prime 2}}{2 B \rho}+\frac{B^{\prime \prime}}{\rho}\right) x y^{2}\right]+\hat{\mathbf{x}}\left(\frac{B^{2}}{6 B \rho}+\frac{B^{\prime \prime}}{3 \rho}\right) y^{3} \\
& +\hat{\mathbf{s}}\left[B^{\prime} y-\frac{B^{\prime}}{\rho} x y+\frac{B^{\prime}}{\rho^{2}} x^{2} y-\frac{B^{\prime \prime \prime}}{6} y^{3}\right] .
\end{aligned}
$$

We use formula (1.6):

$$
\mathbf{B}_{B}=\nabla \times \mathbf{A}_{B \mathrm{em}}=\hat{\mathbf{x}} \frac{\partial A_{B \mathrm{em} s}}{\partial y}-\hat{\mathbf{s}} \frac{\partial A_{B x}}{\partial y}+\hat{\mathbf{y}}\left(\frac{1}{1+\rho^{-1} x} \frac{\partial A_{B x}}{\partial s}-\frac{1}{1+\rho^{-1} x} \frac{\partial A_{B s}}{\partial x}\right) .
$$

Comparing Eq. (2.40) with Eq. (2.39), we solve for

$$
\begin{aligned}
\mathbf{A}_{B}= & \hat{\mathbf{s}} \\
& {\left[-B x-\frac{B}{2 \rho} x^{2}+\left(\frac{B^{2}}{24 B \rho}+\frac{B^{\prime \prime}}{12 \rho}\right) y^{4}\right] } \\
& +\hat{\mathbf{x}}\left[-\frac{B^{\prime}}{2} y^{2}+\frac{B^{\prime}}{2 \rho} x y^{2}-\frac{B^{\prime}}{2 \rho^{2}} x^{2} y^{2}+\frac{B^{\prime \prime \prime}}{24} y^{4}\right] .
\end{aligned}
$$

To get the gradient part of the field

$$
\mathbf{B}_{G 1}=\hat{\mathbf{y}} G(s) x,
$$

we proceed as above, starting with the potential

$$
\psi_{G 2}=G(s) x y
$$

Formula (1.4) gives

$$
\nabla^{2} \psi_{G 2}=\frac{G y}{\rho}+\left(G^{\prime \prime}-\frac{G}{\rho^{2}}\right) x y+\cdots
$$

so we add the terms:

$$
\psi_{G 4}=G(s) x y-\frac{G y^{3}}{6 \rho}+\left(\frac{G}{6 \rho^{2}}-\frac{G^{\prime \prime}}{6}\right) x y^{3} .
$$

There are no more fourth-order terms. The field is, if we use Eq. (1.3),

$$
\begin{aligned}
& \mathbf{B}_{G}= \nabla \psi_{G 4} \\
&= \hat{\mathbf{y}}\left[G x-\frac{G y^{2}}{2 \rho}+\left(\frac{G}{2 \rho^{2}}-\frac{G^{\prime \prime}}{2}\right) x y^{2}\right]+\hat{\mathbf{x}}\left[G y+\left(\frac{G}{6 \rho^{2}}-\frac{G^{\prime \prime}}{6}\right) y^{3}\right] \\
&+\hat{\mathbf{s}}\left[G^{\prime} x y-\frac{G^{\prime}}{\rho} \frac{x^{2} y}{\rho}-\frac{G^{\prime} y^{3}}{6 \rho}\right] . \\
& 8
\end{aligned}
$$


We use formula (1.6) as above and solve for

$$
\begin{aligned}
\mathbf{A}_{G}= & \hat{\mathbf{s}}\left[\frac{G}{2}\left(y^{2}-x^{2}\right)-\frac{G}{3 \rho} x^{3}+\frac{G}{2 \rho} x y^{2}+\left(\frac{G}{24 \rho^{2}}-\frac{G^{\prime \prime}}{24}\right) y^{4}\right] \\
& +\hat{\mathbf{x}}\left[-\frac{G^{\prime}}{2} x y^{2}+\frac{G^{\prime}}{2 \rho} x^{2} y^{2}+\frac{G^{\prime}}{24 \rho} y^{4}\right] .
\end{aligned}
$$

We combine Eqs. (2.41) and (2.47) to get the total canonical magnetic potential:

$$
\begin{aligned}
\mathbf{A}= & \mathbf{A}_{B}+\mathbf{A}_{G} \\
= & \hat{\mathbf{s}}\left[-B x-\left(\frac{B}{2 \rho}+\frac{G}{2}\right) x^{2}+\frac{G}{2} y^{2}-\frac{G}{3 \rho} x^{3}+\frac{G}{2 \rho} x y^{2}+\right. \\
& \left.\left(\frac{B^{\prime 2}}{24 B \rho}+\frac{B^{\prime \prime}}{12 \rho}-\frac{G^{\prime \prime}}{24}+\frac{G}{24 \rho^{2}}\right) y^{4}\right] \\
& +\hat{\mathbf{x}}\left[-\frac{B^{\prime}}{2} y^{2}+\left(\frac{B^{\prime}}{2 \rho}-\frac{G^{\prime}}{2}\right) x y^{2}-\left(\frac{B^{\prime}}{2 \rho^{2}}-\frac{G^{\prime}}{2 \rho}\right) x^{2} y^{2}+\left(\frac{B^{\prime \prime \prime}}{24}+\frac{G^{\prime}}{24 \rho}\right) y^{4}\right] .
\end{aligned}
$$

Formula (2.48), if we put $G=0$, agrees with formula (2.31) in the interior of the magnet, as it must, but not all the edge terms agree. Because the reference orbit in the edge is essentially straight for a thin edge perpendicular to the reference orbit, one would expect these two formulas to agree in this limit. The problem is that the edge terms depend on $B^{\prime}$ and $B^{\prime \prime}$, and these derivatives go to infinity in the limit of a thin edge. It therefore makes a difference whether we regard the edge as straight as in formula (2.31) or as following the curved reference orbit as in formula (2.48), even in the thin edge limit. This is one of those mathematical oddities where the limiting case is not equal to the limit approached by the nonlimiting case. Of course in the limit, the differences are confined to an infinitesimal distance along the reference orbit, but the edge fields are also large, so they can have an effect on the particle orbit.

\subsection{Quadrupole}

We specify a quadrupole by giving the field in the neighborhood of the reference orbit:

$$
\mathbf{B}=\hat{\mathbf{y}} Q(s) x+\hat{\mathbf{x}} Q(s) y .
$$

The field on the axis of a quadrupole is zero, so the reference orbit is a straight line. The accelerator coordinate system $x, y, s$ is a rectangular coordinate system. Let us use polar coordinates in the $x, y$ plane:

$$
x=r \sin \theta, y=r \cos \theta .
$$

The magnetic scalar potential near the reference orbit that gives the field $(2.49)$ is

$$
\psi_{o}(x, y, s)=Q(s) x y=\frac{1}{2} Q(s) r^{2} \sin 2 \theta .
$$

We need the potential to fourth order in $r$. In order to specify a particular solution of Laplace's equation, we will require as a boundary condition that the quadrupole magnet, including the ends, have quadrupole symmetry and have the proper symmetry in the median plane. The magnetic scalar potential is then proportional to $\sin 2 \theta$ :

$$
\psi(r, \theta, s)=\sum_{n} \psi_{n}(s) r^{n} \sin 2 \theta
$$


Laplace's equation is

$$
\begin{aligned}
\nabla^{2} \psi & =\frac{1}{r} \frac{\partial}{\partial r} r \frac{\partial \psi}{\partial r}+\frac{1}{r^{2}} \frac{\partial^{2} \psi}{\partial \theta^{2}}+\frac{\partial^{2} \psi}{\partial s^{2}} \\
& =\sum_{n}\left[\psi_{n}^{\prime \prime}+\left[(n+2)^{2}-4\right] \psi_{n+2}\right] r^{n} \sin 2 \theta=0
\end{aligned}
$$

which gives

$$
\psi_{n+2}=-\frac{\psi_{n}^{\prime \prime}}{(n+2)^{2}-4}
$$

or conversely

$$
\psi_{n-2}^{\prime \prime}=-\left(n^{2}-4\right) \psi_{n} .
$$

Given $\psi_{n}, n>0$, Eq. (2.54) gives us $\psi_{n+2}, \psi_{n+4}, \ldots$ Conversely, given $\psi_{n}, n>2$, Eq. (2.55) gives us $\psi_{n-2}, \psi_{n-4}, \ldots$, terminating at $\psi_{2}$ if $n$ is even. We do not allow $n$ odd, because negative powers of $r$ misbehave at the origin. The solution therefore begins with $\psi_{2}=Q / 2$. The sum is over even powers of $r$ and is therefore a sum of polynomials in $x, y$. To fourth order it is

$$
\psi=Q(s) x y-\frac{Q^{\prime \prime}}{12}\left(x^{3} y+x y^{3}\right) \text {. }
$$

The field is, to third order

$$
\mathbf{B}=\hat{\mathbf{x}}\left[Q y-\frac{Q^{\prime \prime}}{12}\left(3 x^{2} y+y^{3}\right)\right]+\hat{\mathbf{y}}\left[Q x-\frac{Q^{\prime \prime}}{12}\left(x^{3}+3 x y^{2}\right)\right]+\hat{\mathbf{s}} Q^{\prime} x y,
$$

which can be derived from the vector potential

$$
\mathbf{A}=\hat{\mathbf{s}}\left[\frac{Q}{2}\left(y^{2}-x^{2}\right)+\frac{Q^{\prime \prime}}{48}\left(x^{4}-6 x^{2} y^{2}-y^{4}\right)\right]-\hat{\mathbf{x}} \frac{Q^{\prime}}{2} x y^{2} .
$$

\subsection{Skew Quadrupole}

We may need the potential for a skew quadrupole, either to represent a small error in a magnet or misalignment in a quadrupole, or for a lattice element whose purpose is to correct for such errors. We proceed as above, specifying the skew quadrupole by the field in the neighborhood of the reference orbit:

$$
\mathrm{B}=\hat{\mathbf{x}} Q_{s}(s) x-\hat{\mathbf{y}} Q_{s}(s) y .
$$

The resulting scalar potential has the $\theta$ dependence $\cos 2 \theta$. If we replace $\sin 2 \theta$ in the above discussion by $\cos 2 \theta$, Eq. (2.56) becomes:

$$
\psi=\frac{Q_{s}}{2}\left(x^{2}-y^{2}\right)-\frac{Q_{s}^{\prime \prime}}{24}\left(x^{4}-y^{4}\right)
$$

The field is, to third order

$$
\mathbf{B}=\hat{\mathbf{x}}\left[Q_{s} x-\frac{Q_{s}^{\prime \prime}}{6} x^{3}\right]-\hat{\mathbf{y}}\left[Q_{s} y-\frac{Q_{s}^{\prime \prime}}{6} y^{3}\right]+\hat{\mathbf{s}} \frac{Q_{s}^{\prime}}{2}\left(x^{2}-y^{2}\right),
$$

which can be derived from the vector: potential

$$
\mathbf{A}=\hat{\mathbf{s}}\left[Q_{s} x y-\frac{Q_{s}^{\prime \prime}}{6} x^{3} y\right]+\hat{\mathbf{x}} Q_{s}^{\prime}\left(\frac{y^{3}}{6}-\frac{x^{2} y}{2}\right)
$$


Check of Quadrupole Formulas. We may check the quadrupole formulas by rotating formula (2.62) through $45^{\circ}$. We put

$$
x=\frac{\underline{x}+\underline{y}}{\sqrt{2}}, y=\frac{-\underline{x}+\underline{y}}{\sqrt{2}}, \hat{x}=\frac{\underline{\hat{x}}+\underline{\hat{y}}}{\sqrt{2}}, \hat{y}=\frac{-\underline{\hat{x}}+\underline{\hat{y}}}{\sqrt{2}} .
$$

Formula (2.62) becomes:

$$
\begin{aligned}
\underline{\mathbf{A}}= & \hat{\mathbf{s}}\left[\frac{Q_{s}}{2}\left(\underline{y}^{2}-\underline{x}^{2}\right)+\frac{Q_{s}^{\prime \prime}}{24}\left(\underline{x}^{4}+2 \underline{x}^{3} \underline{y}-2 \underline{x} \underline{y}^{3}-\underline{y}^{4}\right)\right] \\
& +(\underline{\hat{\mathbf{x}}}+\underline{\hat{\mathbf{y}}}) \frac{Q_{s}^{\prime}}{12}\left[\underline{x}^{3}+3 \underline{x}^{2} \underline{y}-3 \underline{x}^{2}-\underline{y}^{3}\right] .
\end{aligned}
$$

We change the gauge via the function

$$
f(x, y, s)=\frac{Q_{s}^{\prime}}{48}\left[\underline{x}^{4}+4 \underline{x}^{3} \underline{y}+6 \underline{x}^{2} \underline{y}^{2}-4 \underline{x} \underline{y}^{3}-\underline{y}^{4}\right] .
$$

We then verify that formula (2.58), if we identify underlined coordinates with the coordinates in Eq. (2.58) and $Q_{s}$ with $Q$, is given by

$$
\mathbf{A}=\underline{\mathbf{A}}-\nabla f
$$

\subsection{Sextupole}

We specify a sextupole by the field near the reference orbit:

$$
\mathbf{B}=\hat{\mathbf{y}} \frac{S(s)}{2}\left(x^{2}-y^{2}\right)+\hat{\mathbf{x}} S(s) x y .
$$

The corresponding potential is

$$
\begin{aligned}
\psi & =\frac{1}{6} S(s) r^{3} \sin 3 \theta \\
& =\frac{1}{6} S(s)\left(3 x^{2} y-y^{3}\right)
\end{aligned}
$$

This gives the field (2.67) and is already third order, so the next term would be fifth order, and we may stop here.

The field to third order is

$$
\mathbf{B}=\hat{\mathbf{y}} \frac{S(s)}{2}\left(x^{2}-y^{2}\right)+\hat{\mathbf{x}} S(s) x y+\hat{\mathbf{s}} \frac{S^{\prime}}{6}\left(3 x^{2} y-y^{3}\right),
$$

which is given by the vector potential

$$
\mathbf{A}=\hat{\mathbf{s}} S\left(\frac{1}{2} x y^{2}-\frac{1}{6} x^{3}\right)+\hat{\mathbf{x}} S^{\prime}\left(-\frac{1}{4} x^{2} y^{2}+\frac{1}{24} y^{4}\right)
$$




\subsection{Octupole}

We specify an octupole by the field near the reference orbit:

$$
\mathbf{B}=\hat{\mathbf{y}} O(3)\left(3 y^{2} x-x^{3}\right)-\hat{\mathbf{x}} O(s)\left(3 x^{2} y-y^{3}\right) .
$$

The corresponding potential is

$$
\begin{aligned}
\psi & =-\frac{1}{4} O(s) r^{4} \sin 4 \theta \\
& =-O(s)\left(x^{3} y-x y^{3}\right)
\end{aligned}
$$

This gives the field (2.71) and is already fourth order, so we may stop here.

The field to third order is just that given by Eq. (2.71). It is given by the vector potential

$$
\mathbf{A}=\hat{\mathbf{s}} O\left(\frac{1}{4} x^{4}-\frac{3}{2} x^{2} y^{2}+\frac{1}{4} y^{4}\right)
$$

\subsection{Accelerating Gap}

We will specify an accelerating gap by the electric field along the reference orbit:

$$
\mathbf{E}=\hat{\mathbf{s}} \Delta(s) V(t),
$$

where $V(t)$ is the gap voltage function, and $\Delta(s)$ is a function whose integral across the gap is 1 and which describes the field shape through the gap; it is usually taken to be simply $\Delta(s)=\delta\left(s-s_{\text {gap }}\right)$. We assume that the gap is formed by two conducting plates with circular holes centered on the reference orbit. Because of the cylindrical symmetry about the $s$ axis, it will be convenient to use cylindrical polar coordinates $r, \theta, s$ as defined by Eq. (2.50).

Because the light transit time across the gap is small compared with other relevant times, it is tempting to derive $\mathbf{E}$ from a static scalar electric potential $\phi$. Because the potential jumps by the gap voltage as we cross the gap, the electric potential cannot be a periodic function of $s$ in a circular machine, and moreover it does not fall to zero outside the region of the gap. We could handle that problem, in analogy with our treatment of the magnetic lattice elements, by deriving the electric field in the gap and then deriving the corresponding vector potential via the relation

$$
\mathbf{E}=-\frac{\partial \mathbf{A}}{\partial t}
$$

However, we will find that all but the main term in our result will correspond to transit time corrections. It is hence questionable whether we can properly neglect the light transit time across the gap. We will therefore work directly with the vector potential, related to the electric field by Eq. (2.75). The field (2.74) on the reference orbit is derived from the vector potential

$$
\mathbf{A}=-\hat{\mathbf{s}} \Delta(s) T(t),
$$

where

$$
T(t)=\int_{0}^{t} V(t) d t
$$

and the lower limit of the integral is arbitrary. It is convenient to use the Lorentz gauge so that the potentials satisfy the wave equation. The Lorentz gauge is defined by

$$
\nabla \cdot \mathbf{A}-\frac{1}{c} \frac{\partial \phi}{\partial t}=0
$$


Because the electric potential $\phi$ vanishes on the reference orbit, it will be zero everywhere, and we will omit it from our discussion.

The vector potential in cylindrical coordinates may be written:

$$
\mathbf{A}=\hat{\mathbf{r}}(\theta) A_{r}(r, s, t)+\hat{\theta}(\theta) A_{\theta}(r, s, t)+\hat{\mathbf{s}} A_{s}(r, s, t),
$$

where we have indicated coordinate dependences explicitly to exhibit the cylindrical symmetry. We expand the components in power series in $r$ :

$$
\begin{aligned}
& A_{r}=\sum_{n} a_{r n}(s, t) r^{n} \\
& A_{\theta}=\sum_{n} a_{\theta n}(s, t) r^{n} \\
& A_{s}=\sum_{n} a_{s n}(s, t) r^{n}
\end{aligned}
$$

Because there are no charges or currents in the gap (except those of the beam which are treated later), the vector potential satisfies the wave equation:

$$
\begin{aligned}
\nabla^{2} \mathbf{A}-\frac{1}{c^{2}} \frac{\partial^{2} \mathbf{A}}{\partial t^{2}}= & 0 \\
= & \hat{\mathbf{r}} \sum_{n}\left[n^{2} a_{r n} r^{n-2}-a_{r n} r^{n-2}+a_{r n}^{\prime \prime} r^{n}-c^{-2} \ddot{a}_{r n} r^{n}\right] \\
& +\hat{\theta} \sum_{n}\left[n^{2} a_{\theta n} r^{n-2}-a_{\theta n} r^{n-2}+a_{\theta n}^{\prime \prime} r^{n}-c^{-2} \ddot{a}_{\theta n} r^{n}\right] \\
& +\hat{\mathbf{s}} \sum_{n}\left[n^{2} a_{s n} r^{n-2}+a_{s n}^{\prime \prime} r^{n}-c^{-2} \ddot{a}_{s n} r^{n}\right]
\end{aligned}
$$

Dots denote derivatives with respect to $t$. Note that in applying the operator $\nabla^{2}[\mathrm{Eq} .(2.53)]$ to $\mathbf{A}$, we have to differentiate also the unit vectors $\hat{\mathbf{r}}, \hat{\theta}$.

From the $\hat{\mathbf{r}}$ terms in Eq. (2.81) we get

$$
a_{r, n+2}=\frac{-a_{r n}^{\prime \prime}+c^{-2} \ddot{a}_{r n}}{(n+2)^{2}-1}, a_{r, n-2}^{\prime \prime}-c^{-2} \ddot{a}_{r, n-2}=-\left(n^{2}-1\right) a_{r n} .
$$

The first of these equations gives us for any coefficient $a_{r n}$ a coefficient $a_{r, n+2}$. The second of these equations gives us, for any coefficient $a_{r n}$, a coefficient $a_{r, n-2}$, unless $n=1$. Because $n<0$ is not allowed, we conclude that the series for $A_{r}$ begins with $n=1$ and contains terms with all odd values of $n$. A similar conclusion applies to $A_{\theta}$. For $A_{s}$, we have

$$
a_{s, n+2}=\frac{-a_{s n}^{\prime \prime}+c^{-2} \ddot{a}_{s n}}{(n+2)^{2}}, a_{s, n-2}^{\prime \prime}-c^{-2} \ddot{a}_{s, n-2}=-n^{2} a_{s n}
$$

As above, we conclude that the series for $A_{s}$ begins with $n=0$ and contains terms with all even values of $n$. Had we considered the electric potential $\phi$, we would have gotten the same result for the series. Because the term $n=0$ would be zero as we saw above, the entire series for $\phi$ would vanish.

Because $\nabla \cdot \mathbf{E}=0$, we have

$$
\nabla \cdot \mathbf{A}=0=\sum_{n} n a_{r n} r^{n-1}+a_{r n} r^{n-1}+a_{s}^{\prime} r^{n}
$$


from which we get

$$
a_{r, n+1}=-\frac{a_{s n}^{\prime}}{n+2}
$$

so that $\mathbf{A}_{r}$ is determined by $\mathbf{A}_{s}$. By substituting from Eq. (2.83) into Eq. (2.85), we can verify that Eq. (2.82) holds.

From Eq. (2.76), we have

$$
a_{s 0}=-\Delta(s) T(t),
$$

from which, according to the above equations, we get $A_{s}$ and $A_{r}$, which are to fourth order:

$$
\begin{aligned}
& A_{s}=-\Delta T+\frac{\Delta^{\prime \prime} T-\Delta c^{-2} \ddot{T}}{4} r^{2}-\frac{\Delta^{\prime \prime \prime} T-2 \Delta^{\prime \prime} c^{-2} \ddot{T}+\Delta c^{-4} T^{(4)}}{64} r^{4}, \\
& A_{r}=\frac{\Delta^{\prime} T}{2} r-\frac{\Delta^{\prime \prime \prime} T-\Delta^{\prime} c^{-2} \ddot{T}}{16} r^{3}
\end{aligned}
$$

where

$$
T^{(4)}=\frac{d^{4} T}{d t^{4}}
$$

We will set $A_{\theta}=0$, because we do not expect that the gap will produce an electric field $E_{\theta}$ circling the reference orbit. We note that these equations imply a magnetic field in the gap:

$$
\mathbf{B}=\nabla \times \mathbf{A}=\hat{\theta}\left[\frac{\Delta \ddot{T}}{2 c^{2}} r-\frac{\Delta^{\prime \prime} c^{-2} \ddot{T}-\Delta c^{-4} T^{(4)}}{16} r^{3}\right]
$$

The position vector in the $x y$ plane is

$$
\mathbf{r}_{\perp}=\hat{\mathbf{r}} r=\hat{\mathbf{x}} x+\hat{\mathbf{y}} y
$$

and the vector potential for an accelerating gap is

$$
\begin{aligned}
\mathbf{A}= & -\hat{\mathbf{s}} \Delta(s) \int_{0}^{t} V(t) d t \\
& +\hat{\mathbf{s}}\left[\frac{\Delta^{\prime \prime} T-\Delta c^{-2} \ddot{T}}{4}\left(x^{2}+y^{2}\right)-\frac{\Delta^{\prime \prime \prime} T-2 \Delta^{\prime \prime} c^{-2} \ddot{T}+\Delta c^{-4} T^{(4)}}{64}\left(x^{2}+y^{2}\right)^{2}\right] \\
& +\hat{\mathbf{x}}\left[\frac{\Delta^{\prime} T}{2} x-\frac{\Delta^{\prime \prime \prime} T-\Delta^{\prime} c^{-2} \ddot{T}}{16}\left(x^{3}+x y^{2}\right)\right] \\
& +\hat{\mathbf{y}}\left[\frac{\Delta^{\prime} T}{2} y-\frac{\Delta^{\prime \prime \prime} T-\Delta^{\prime} c^{-2} \ddot{T}}{16}\left(x^{2} y+y^{3}\right)\right]
\end{aligned}
$$

The terms in $x, y$ are edge field corrections appropriate to a circular gap perpendicular to the reference orbit; they are usually neglected. The terms involving derivatives of $\Delta$ integrate to zero across the gap, so they involve transit time effects. If $\Delta$ approximates a $\delta$-function, then its $n^{\text {th }}$ derivative with respect to $s$, in an integral across the gap, produces $(-1)^{n}$ times the $n^{\text {th }}$ derivative of the rest of the integrand.

The terms involving time derivatives of $T$ can be evaluated by taking

$$
V(t)=\hat{V} \sin \omega_{\mathrm{rf}} t
$$


where we may neglect the time dependence of $\hat{V}$ and $\omega_{\mathrm{rf}}$, because these terms are very small anyway. We then have

$$
\begin{aligned}
T & =-\frac{\hat{V}}{\omega_{\mathrm{rf}}} \cos \omega_{\mathrm{rf}} t, \\
c^{-2} \ddot{T} & =-\frac{\omega_{\mathrm{rf}}^{2}}{c^{2}} T, \\
c^{-4} T^{(4)} & =\frac{\omega_{\mathrm{rf}}^{4}}{c^{4}} T .
\end{aligned}
$$

For a circular accelerator, the $s$ derivative of $x$ or $y$ is typically $\nu / R$ times $x$ or $y$, where $\nu$ is the number of betatron oscillations per revolution and $2 \pi R$ is the circumference. For a harmonic number $h, \omega_{\mathrm{rf}}=h \beta c / R$, so the terms involving $T^{(n)}$ typically differ from those involving $\Delta^{(n)}$ by a factor of about $(h \beta / \nu)^{n}$.

\subsection{Space-Charge Potentials}

In working out space-charge potentials, we will make the standard assumption that the scale lengths along the reference orbit for changes in the beam intensity and cross section and for changes in the vacuum chamber are much larger than the cross-sectional dimensions of the beam or the vacuum chamber. We therefore treat the reference orbit as straight, with a beam and vacuum chamber uniform in $s$.

The electromagnetic potentials satisfy the inhomogeneous wave equations:

$$
\begin{aligned}
\nabla^{2} \mathbf{A}_{\mathrm{em}}-\frac{1}{c^{2}} \frac{\partial^{2} \mathbf{A}_{\mathrm{em}}}{\partial t^{2}} & =-\mu_{0} \mathbf{J}_{b}, \\
\nabla^{2} \phi-\frac{1}{c^{2}} \frac{\partial^{2} \phi}{\partial t^{2}} & =-\frac{\rho_{b}}{\epsilon_{0}},
\end{aligned}
$$

where the terms on the right involve the charge and current densities of the beam. By our assumption, the operator $\nabla^{2}$ differentiates only the cross-sectional variations of the beam and is of order $L_{t}^{-2}$, where $L_{t}$ is a typical transverse dimension of the beam. The time variations are due to the translation of the longitudinal variations along the beam, so the operator $c^{-2} \partial^{2} / \partial t^{2}$ is of order $\left(\beta / L_{\ell}\right)^{2}$, where $L_{\ell}$ is a typical distance for density variations along the beam. Hence we may neglect the time derivatives in Eqs.(2.95), which are at most of order $\left(L_{t} / L_{\ell}\right)^{2}$ relative to the space derivatives.

The beam current is due to its longitudinal velocity. We will neglect any transverse motions of the beam. The current is then

$$
\mathbf{J}_{b}=\hat{\mathbf{s}} \beta c \rho_{b} .
$$

We need to solve the equations

$$
\begin{aligned}
\left(\frac{\partial^{2}}{\partial x^{2}}+\frac{\partial^{2}}{\partial y^{2}}\right) \mathbf{A}_{\mathrm{em}} & =-\mu_{0} \hat{\mathbf{s}} \beta c \rho_{b}=-\hat{\mathbf{s}} \frac{\beta}{c} \frac{\rho_{b}}{\epsilon_{0}} \\
\left(\frac{\partial^{2}}{\partial x^{2}}+\frac{\partial^{2}}{\partial y^{2}}\right) \phi & =-\frac{\rho_{b}}{\epsilon_{0}} .
\end{aligned}
$$

We now assume that the boundary condition on $\mathbf{A}_{\mathrm{em}}$ is the same as that on $\phi$, for example that they both vanish at the vacuum chamber wall, which will be true if the vacuum chamber is a perfect conductor. If that is so, then we conclude that

$$
\mathbf{A}_{\mathrm{em}}=\hat{\mathbf{s}} \frac{\beta}{c} \phi .
$$


This assumption is often not true, in which case Eqs. (2.97) and (2.98) must be solved separately. (See Laslett [9] for details.)

An electron beam may be fully or partially neutralized by stationary positive ions. If the fractional neutralization is $f$, i.e., if the ion charge density is $-f \rho_{b}$, then the charge density in Eq. (2.98), but not the current density, must be reduced by a factor $(1-f)$. Note that this assumes that the neutralizing charge distribution is the same as that of the beam, which should be approximately true if the beam density is not rapidly changing. It is probably not true for a bunched beam, because the ions will not move rapidly enough to adjust their density to that of the beam; in this case, the ion density will probably have the distribution of the average beam density, averaged over a revolution. The electrostatic potential of the ion beam would then have to be calculated separately and added to the electric potential of the beam.

Let the beam charge density be given by

$$
\rho_{b}=\lambda h(x, y)
$$

where $\lambda$ is the charge per unit length, and $h(x, y)$ is its cross-sectional distribution, normalized so that its integral over the vacuum chamber cross section is one. The charge density $\lambda$ and the distribution $h(x, y)$ are slowly varying functions of $s$ and $t$. The solution of Eq. (2.98) for $\phi$ is

$$
\phi(x, y)=\frac{(1-f) \lambda}{4 \pi \epsilon_{0}} g(x, y)
$$

where $g(x, y)$ is the solution of

$$
\left(\frac{\partial^{2}}{\partial x^{2}}+\frac{\partial^{2}}{\partial y^{2}}\right) g(x, y)=-4 \pi h(x, y)
$$

For a uniform charge density in a circular cross section of radius $a$, centered in a circular perfect conductor of radius $b$, we have

$$
h(x, y)= \begin{cases}\frac{1}{\pi a^{2}} & \text { for } x^{2}+y^{2} \leq a^{2} \\ 0 & \text { for } a^{2}<x^{2}+y^{2} \leq b^{2} .\end{cases}
$$

We can solve Eq. (2.102) most easily using polar coordinates. By symmetry, neither $h$ nor $g$ depends on $\theta$, so Eq. (2.102) becomes:

$$
\frac{1}{r} \frac{d}{d r} r \frac{d g}{d r}=-4 \pi h
$$

Equation (2.104) is easily integrated twice, using Eq. (2.103) for $h$. We note that $d g / d r$ vanishes at $r=0$, which fixes the first arbitrary constant. We require that $g$ vanish at $r=b$, which fixes the remaining arbitrary constant. The result is, in rectangular coordinates:

$$
g(x, y)= \begin{cases}1+2 \ln \frac{b}{a}-\frac{x^{2}+y^{2}}{a^{2}} & \text { if } x^{2}+y^{2} \leq a^{2}, \\ \ln \frac{b^{2}}{x^{2}+y^{2}} & \text { if } a^{2} \leq x^{2}+y^{2} \leq b^{2} .\end{cases}
$$

The radius $a$ (also $b$ ) may be a slowly varying function of $s$ and perhaps also of $t$. For a different but still circularly symmetric distribution $h(x, y)$, the result would not be much different. For example, for the parabolic distribution

$$
h(x, y)= \begin{cases}\frac{2}{\pi a^{2}}\left(1-\frac{x^{2}+y^{2}}{a^{2}}\right) & \text { for } x^{2}+y^{2} \leq a^{2}, \\ 0 & \text { for } a^{2}<x^{2}+y^{2} \leq b^{2}\end{cases}
$$


we get

$$
g(x, y)= \begin{cases}\frac{3}{2}+2 \ln \frac{b}{a}-\frac{2\left(x^{2}+y^{2}\right)}{a^{2}}+\frac{\left(x^{2}+y^{2}\right)^{2}}{2 a^{4}} & \text { if } x^{2}+y^{2} \leq a^{2}, \\ \ln \frac{b^{2}}{x^{2}+y^{2}} & \text { if } a^{2} \leq x^{2}+y^{2} \leq b^{2} .\end{cases}
$$

Some other cases are treated in the literature; see Refs. [1, 2, 9].

The canonical potentials for space-charge fields are

$$
\begin{aligned}
\phi & =\frac{(1-f) \lambda}{4 \pi \epsilon_{0}} g(x, y) \\
\mathbf{A} & =\hat{\mathbf{s}} \frac{\beta \lambda}{4 \pi \epsilon_{0} c}\left(1+\rho^{-1} x\right) g(x, y) .
\end{aligned}
$$

As written in Eqs. (2.108) and (2.109), the potentials $\phi$ and A depend explicitly only on the variables $x$ and $y$. However, the charge density $\lambda$ and perhaps also $g(x, y)$ will generally depend also on $s$ and $t$. In a beam traveling with a velocity $\beta c$, the charge density will be approximately $\lambda(s-\beta c t)$, if we neglect the slow changes in density in the beam frame of reference.

\section{TRANSVERSE MOTION}

We start with Eq. (1.11) and delete the electric potential $\phi$ to obtain the Hamiltonian for transverse motion:

$$
H_{s T 1}\left(x, \underline{p_{x}}, y, \underline{p_{y}} ; s\right)=-e A_{s}-\left(1+\rho^{-1} x\right)\left[p^{2}-\left(\underline{p_{x}}-e A_{x}\right)^{2}-\underline{p_{y}^{2}}\right]^{\frac{1}{2}},
$$

where the kinetic momentum is

$$
p=\left[\frac{E^{2}-m^{2} c^{4}}{c^{2}}\right]^{\frac{1}{2}}
$$

and we are using a gauge in which $A_{y}=0$. We have underlined the momenta, because we want to rescale them.

For each particle and for pure transverse motion, the kinetic momentum $p$ is a constant parameter. It is convenient to rescale the canonical momenta to be $1 / p$ (not $1 / p_{0}$ ) times their values in Eq. (3.1):

$$
p_{x}=\underline{p_{x}} / p, p_{y}=\underline{p_{y}} / p .
$$

This makes the kinetic part of these momenta equal to the slopes $d x / d s, d y / d s$. The reader may verify that this requires also rescaling the Hamiltonian by $1 / p$ :

$$
\begin{aligned}
H_{s T}\left(x, p_{x}, y, p_{y} ; s\right) & =H_{s T 1} / p \\
& =-\frac{e A_{s}}{p}-\left(1+\rho^{-1} x\right)\left[1-\left(p_{x}-\frac{e A_{x}}{p}\right)^{2}-p_{y}^{2}\right]^{\frac{1}{2}}
\end{aligned}
$$

\subsection{Space-Charge Terms}

The space-charge terms include the electric potential (2.108), which can be included here in the following way. The space-charge force on a particle is given in terms of the space-charge fields by

$$
\begin{aligned}
\mathbf{F} & =e \mathbf{E}+e \mathbf{v} \times \mathbf{B} \\
& =-e \nabla \phi-e \frac{\partial \mathbf{A}_{\mathrm{em}}}{\partial t}+e c \beta \hat{s} \times\left(\nabla \times \mathbf{A}_{\mathrm{em}}\right)
\end{aligned}
$$


where we neglect the transverse components of the velocity, which would make only a very small correction to the space-charge force. According to Eq. (2.109), the space-charge vector potential has only an $s$ component $\left(\mathbf{A}_{\mathrm{em}}=\mathfrak{s} A_{\mathrm{ems}}\right)$. The vector potential part of the space-charge force is therefore:

$$
\begin{aligned}
-e \frac{\partial \mathbf{A}_{\mathrm{em}}}{\partial t}+e c \beta \hat{s} \times\left(\nabla \times \mathbf{A}_{\mathrm{em}}\right) & =-e \hat{s} \frac{\partial A_{\mathrm{em} s}}{\partial t}+e c \beta \hat{s} \times\left(\nabla A_{\mathrm{em} s} \times \hat{s}\right) \\
& =-e \hat{s} \frac{\partial A_{\mathrm{em} s}}{\partial t}+e c \beta \nabla A_{\mathrm{em} s}-e c \beta \hat{s} \frac{\partial A_{\mathrm{em} s}}{\partial s}
\end{aligned}
$$

If we neglect transverse velocity components,

$$
\frac{\partial A_{\mathrm{em} s}}{\partial t}=\frac{\partial A_{\mathrm{em} s}}{\partial s} \frac{d s}{d t}=\beta_{w} c \frac{\partial A_{\mathrm{em} s}}{\partial s},
$$

where $\beta_{w} c$ is the velocity of whatever wave disturbance on the beam is producing $\partial A_{\mathrm{em} s} / \partial t$. If we approximate $\beta_{w}$ by $\beta$, then two terms in Eq. (3.6) cancel out, leaving

$$
-e \frac{\partial \mathbf{A}_{\mathrm{em}}}{\partial t}+e c \beta \hat{s} \times\left(\nabla \times \mathbf{A}_{\mathrm{em}}\right)=+e c \beta \nabla A_{\mathrm{em} s} .
$$

We substitute in Eq. (3.5):

$$
\mathbf{F}=-e \nabla\left(\phi-\beta c A_{\mathrm{em} s}\right)=e \beta c \nabla A_{\mathrm{emscs}},
$$

where we have used Eqs. (2.108) and (2.109) and set

$$
A_{\mathrm{emscs}}=-\left(\frac{1}{\gamma^{2}}-f\right) \frac{\lambda}{4 \pi \epsilon_{0} c \beta} g(x, y) \text {. }
$$

By comparing this result with Eq. (3.8), we see the space-charge force can be included in the Hamiltonian (3.4) by including in $A_{s}$ the canonical potential

$$
A_{\mathrm{scs}}=-\left(1+\rho^{-1} x\right)\left(\frac{1}{\gamma^{2}}-f\right) \frac{\lambda}{4 \pi \epsilon_{0} c \beta} g(x, y)
$$

which is not the true space-charge canonical potential, but which gives the correct space-charge force including the $\phi$ term, under the conditions for the validity of Eqs. (2.108), (2.109), and (3.8).

\subsection{Fourth-Order Terms}

The expansion of Eq. (3.4) to fourth order in $x, y, p_{x}, p_{y}$ is, if we use the formulas for $\mathbf{A}$ derived in section 2:

$$
\begin{aligned}
H_{s T}= & \frac{1}{2} p_{x}^{2}+\frac{1}{2} p_{y}^{2}-\frac{x}{\rho}\left(1-\frac{p_{0}}{p}\right)-\sum_{k+\ell=2}^{4} \frac{e A_{s k \ell}}{p} x^{k} y^{\ell} \\
& -\frac{e A_{x 02}}{p} y^{2} p_{x}+\frac{x\left(p_{x}^{2}+p_{y}^{2}\right)}{2 \rho} \\
& +\frac{e^{2} A_{x 02}^{2}}{2 p^{2}} y^{4}-\frac{e A_{x 21}}{p} x^{2} y p_{x}-\left(\frac{e A_{x 02}}{p \rho}+\frac{e A_{x 12}}{p}\right) x y^{2} p_{x}-\frac{e A_{x 03}}{p} y^{3} p_{x}+\frac{1}{8}\left(p_{x}^{2}+p_{y}^{2}\right)^{2} \\
& +C_{s c}\left(x^{2}+y^{2}\right)+\frac{C_{s c}}{\rho}\left(x^{3}+x y^{2}\right)
\end{aligned}
$$


where $p_{0}$ is the momentum of the reference particle given by Eq. (1.1), the coefficients $A_{s k \ell}$ and $A_{x k \ell}$ are the coefficients multiplying $x^{k} y^{\ell}$ in the appropriate terms of Eqs. (2.30), (2.48), (2.58), $(2.62),(2.70)$, and (2.73), and

$$
C_{s c}=\left(\frac{1}{\gamma^{2}}-f\right) \frac{e \lambda}{4 \pi \epsilon_{0} a^{2} \beta p}
$$

is the coefficient of the space-charge terms, if we use Eq. (2.105). Terms independent of the coordinates have been dropped from Eq. (3.12).

\section{LONGITUDINAL MOTION}

In this section, we obtain the one-dimensional Hamiltonian for longitudinal motion, to lowest order in the energy deviation, in several convenient forms. We derive the Hamiltonian by writing down the equations of motion and writing the Hamiltonian by inspection. Our treatment follows in part that of Reference [1]. We first treat the case where the rf frequency $\omega_{\mathrm{rf}}$ is constant, applicable particularly to a linac or storage ring. We next allow $\omega_{\mathrm{rf}}$ to depend on the time, as in a circular accelerator.

Section 5 contains the derivation of the complete Hamiltonian for nonlinear coupled horizontal and longitudinal motion, starting from the original Hamiltonian (1.11) and making successive canonical transformations to synchrotron, betatron variables.

\subsection{Second-Order Hamiltonian with Constant Radio Frequency}

We will specify the accelerating gap(s) as in Eq. (2.74) by the functions $\Delta(s)$ and $V(t)$, which we take to be:

$$
\begin{aligned}
\Delta(s) & =\sum_{j} \delta\left(s-s_{j}\right), \\
V(t) & =\hat{V} \sin \omega_{\mathrm{rf}} t
\end{aligned}
$$

where we will assume in this subsection that $\omega_{\text {rf }}$ is constant. This is appropriate for a linac, where $\omega_{\mathrm{rf}}$ is at least piecewise constant, and for a storage ring. We may neglect the time variation of $\omega_{\mathrm{rf}}$ in a circular accelerator for short times, for example to draw bucket diagrams and obtain bucket parameters. The voltage $\hat{V}$ may depend on $j$ or, equivalently, on $s$.

The gap positions are separated by distances $L$ :

$$
L=s_{j+1}-s_{j}
$$

For a circular accelerator with one gap, the gaps are located at $s_{j}=s_{\text {gap }}+2 \pi j R$, where $L=2 \pi R$ is the length of the reference orbit. For a linac, $L$ may be a slowly varying function of $j$ or $s$. Cases in which a circular accelerator has several gaps, or in which a linac has a more complicated gap structure than we have assumed, can be handled in a straightforward way by the methods we will use (both here and in section 5). Treating the general case is not worth the extra complication it would introduce into this report.

Synchrotron Variables. We define the synchronous energy as the energy corresponding to the reference orbit:

$$
E_{s}=\left[p_{0}^{2} c^{2}+m^{2} c^{4}\right]^{1 / 2}
$$


where $p_{0}$ is the momentum corresponding to the reference orbit. We assume the accelerator is designed so that there is a synchronous particle whose energy is the synchronous energy except that it gains energy in discrete increments each time it crosses a gap. It arrives at a gap at a time $t_{s}$ and phase

$$
\omega_{\mathrm{rf}} t_{s}=\varphi_{s}+2 \pi \ell_{j},-\pi<\varphi_{s} \leq \pi .
$$

We include the term with the integer $\ell_{j}$ in order to allow the synchronous phase $\varphi_{s}$ to be constant or at most slowly varying. The energy of the synchronous particle satisfies the equation of motion

$$
\frac{d E_{s}}{d s}=\Delta(s) e \hat{V} \sin \varphi_{s}
$$

This is on average

$$
\left\langle\frac{d E_{s}}{d s}\right\rangle_{\mathrm{av}}=\frac{e \hat{V} \sin \varphi_{s}}{L}
$$

We will neglect the slow variation (if any) of the synchronous phase.

A non-synchronous particle arrives at the gap at a phase

$$
\omega_{\mathrm{rf}} t=\varphi+2 \pi \ell_{j} .
$$

Its energy deviation is

$$
w=E-E_{s} .
$$

We will derive a Hamiltonian for the variables $\varphi, w$ with independent variable $s$.

We first note that because the Poisson bracket

$$
(\varphi, w)_{t,-E}=\frac{\partial \varphi}{\partial t} \frac{\partial w}{\partial(-E)}-\frac{\partial \varphi}{\partial(-E)} \frac{\partial w}{\partial t}=-\omega_{\mathrm{rf}}
$$

is constant, the transformation from $t,-E$ to $\varphi, w$ is canonical with scale factor $-\omega_{\mathrm{rf}}$, and the variables $\varphi, w$ are canonical. In particular,

$$
d \varphi d w=\omega_{\mathrm{rf}} d t d E
$$

and because Liouville's theorem holds in the $t, E$ plane, it will hold also in the $\varphi, w$ plane.

The Hamiltonian. A particle gains energy $\hat{V} \sin \varphi$ when it crosses a gap. Its energy equation of motion is therefore:

$$
\frac{d w}{d s}=\Delta(s) e \hat{V}\left(\sin \varphi-\sin \varphi_{s}\right)
$$

The time for the synchronous particle to travel between gaps is

$$
\tau=\frac{L}{\beta c} \text {. }
$$

For an off-momentum particle, we will define the parameter $\eta$ by writing to first order

$$
\frac{\Delta \tau}{\tau}=\eta \frac{\Delta p}{p}
$$

The change of phase, relative to the synchronous particle whose phase is not changing, is

$$
\begin{aligned}
\Delta \varphi & =\omega_{\mathrm{rf}} \Delta \tau \\
& =\omega_{\mathrm{rf}} \tau \eta \frac{\Delta p}{p} \\
& =\frac{\omega_{\mathrm{rf}} \tau \eta w}{m c^{2} \beta^{2} \gamma}
\end{aligned}
$$


The phase equation of motion is therefore

$$
\frac{d \varphi}{d s}=\frac{\Delta \varphi}{L}=K w
$$

where

$$
K=\frac{\omega_{\mathrm{rf}} \eta}{c \beta^{3} E_{s}}
$$

and we have used Eq. (4.13). Because Eqs. (4.16) and (4.12) give $d \varphi / d s$ in terms of $w$, and $d w / d s$ in terms of $\varphi$, it is easy to write the corresponding Hamiltonian:

$$
H_{s L}(\varphi, w ; s)=\frac{1}{2} K w^{2}+\Delta(s) e \hat{V}\left(\cos \varphi+\varphi \sin \varphi_{s}\right)
$$

From Eq. (4.13) we have, for a linac:

$$
\begin{aligned}
\frac{\Delta \tau}{\tau} & =-\frac{\Delta \beta}{\beta} \\
& =-\frac{1}{\gamma^{2}} \frac{\Delta(\beta \gamma)}{\beta \gamma} \\
& =-\frac{1}{\gamma^{2}} \frac{\Delta p}{p}
\end{aligned}
$$

where we used $\gamma^{2}=1 /\left(1-\beta^{2}\right)$. So for a linac,

$$
\eta=-\frac{1}{\gamma^{2}}
$$

Here $\gamma$ refers to the synchronous energy, so $\eta$ is a function of $s$. For a circular accelerator, $L$ along the equilibrium orbit is a function of $p$, and

$$
\frac{\Delta \tau}{\tau}=\frac{\Delta L}{L}-\frac{\Delta \beta}{\beta}
$$

Now define the transition energy $\gamma_{t} m c^{2}$ by

$$
\frac{\Delta L}{L}=\frac{1}{\gamma_{t}^{2}} \frac{\Delta p}{p}
$$

where $\Delta L$ is the change in $L$ for an off-momentum particle. Then for a circular accelerator,

$$
\eta=\frac{1}{\gamma_{t}^{2}}-\frac{1}{\gamma^{2}}
$$

Difference Equations of Motion. We note that the equations of motion (4.16) and (4.12), which follow from the Hamiltonian (4.18), imply the difference equations:

$$
\begin{aligned}
\varphi_{j+1} & =\varphi_{j}+\left(s_{j+1}-s_{j}\right) K w_{j}, \\
w_{j+1} & =w_{j}+e \hat{V}\left(\sin \varphi_{j+1}-\sin \varphi_{s}\right),
\end{aligned}
$$

where $\varphi_{j}, w_{j}$ are the values of the variables just after crossing the gap at $s_{j}$. 
Smoothed Hamiltonian. If $\hat{V}$ and the distance $L$ between gaps are slowly varying functions of $s$, we may approximate the Hamiltonian (4.18) by replacing $\Delta(s)$ by its average value:

$$
H_{s L S}(\varphi, w ; s)=\frac{1}{2} K w^{2}+\frac{e \hat{V}}{L}\left(\cos \varphi+\varphi \sin \varphi_{s}\right)-\left(\frac{1}{\gamma^{2}}-f\right) \frac{e \dot{\omega}_{\mathrm{rf}} g_{0}}{4 \pi \epsilon_{0} \beta c} \lambda(\varphi)
$$

The equations of motion resulting from this Hamiltonian do not have any periodically fluctuating coefficient. This feature may be particularly useful in long time simulations, because the $s$ step may now be taken as long as the rate of change of the variables allows.

We have added a space-charge term where $\lambda(\varphi)$ is the charge per unit length at the point in the beam with phase $\varphi$, and

$$
g_{0}=g(0,0)=1+2 \ln \frac{b}{a}
$$

where in the last member, we used Eq. (2.105). The derivation is as follows. According to Eqs. (3.9) and (3.10) the longitudinal force on a particle due to space charge is

$$
F_{s}=-\left(\frac{1}{\gamma^{2}}-f\right) \frac{e g(x, y)}{4 \pi \epsilon_{0}} \frac{\partial \lambda}{\partial s}
$$

where we have neglected any dependence of $g(x, y)$ on $s$. Along the beam, our definitions give

$$
d \varphi=-\frac{\omega_{\mathrm{rf}} d s}{\beta c} .
$$

If we write $\lambda$ as a function of the coordinate $\varphi$, which locates a point on the beam, then we have for the space-charge force on a beam particle

$$
\frac{d w}{d s}=F_{s}=\left(\frac{1}{\gamma^{2}}-f\right) \frac{e \omega_{\mathrm{rf}} g(x, y)}{4 \pi \epsilon_{0} \beta c} \frac{\partial \lambda}{\partial \varphi} .
$$

If we replace $g(x, y)$ by its value at the beam center, then the space-charge force is correctly given by the term we have added to the Hamiltonian (4.26). It would probably be more accurate to replace $g(x, y)$ by its value averaged over the beam cross section. If we do that for a uniform circular beam cross section, the value (4.27) is reduced by a factor $1 / 2$. For a parabolic distribution, the value (4.27) would be reduced by a factor $11 / 12$.

If we use the approximation $d s=\beta c d t$, we can take time as the independent variable and write the smoothed Hamiltonian as

$$
H_{t L S}(\varphi, w ; t)=\frac{1}{2} \beta c K w^{2}+\frac{\epsilon \beta c \hat{V}}{L}\left(\cos \varphi+\varphi \sin \varphi_{s}\right)-\left(\frac{1}{\gamma^{2}}-f\right) \frac{e \omega_{\mathrm{rf}} g_{0}}{4 \pi \epsilon_{0}} \lambda(\varphi) .
$$

For an electron ring, one can include radiation damping by increasing $\varphi_{s}$ above that given by Eq. (4.7) so that the gap provides the extra energy lost per turn by radiation. (See the more careful discussion of this point in section 5.) Radiation loss itself is not described by a Hamiltonian, but here we can bury the loss rate in $\varphi_{s}$.

\subsection{Exact Canonical Variables for Time Varying Radio Frequency}

In a circular accelerator, the radio frequency is a slowly varying function of the time. The variables $\varphi$ and $w$ are then not exactly canonical. If we want Liouville's theorem to hold over long periods of time, for example to study "adiabatic damping," then we need to use variables that are exactly canonical. 
The original longitudinal variables $t$ and $-E$ are exactly canonical. We can construct exactly canonical longitudinal variables as follows. We first take the variation of $\omega_{\mathrm{rf}}$ into account in the definition of $\varphi$ by replacing Eqs. (4.5) and (4.8) by

$$
\begin{gathered}
\int_{0}^{t_{s}} \omega_{\mathrm{rf}}(t) d t=\varphi_{s}+2 \pi \ell_{j},-\pi<\varphi_{s} \leq \pi, \\
\int_{0}^{t} \omega_{\mathrm{rf}}(t) d t=\varphi+2 \pi \ell_{j} .
\end{gathered}
$$

We fix up the Poisson bracket by including $\omega_{\mathrm{rf}}$ in the energy variable:

$$
W=\frac{E-E_{s}}{\omega_{\mathrm{rf}}}
$$

The Poisson bracket is now

$$
(\varphi, W)_{t,-E}=\frac{\partial \varphi}{\partial t} \frac{\partial W}{\partial(-E)}-\frac{\partial \varphi}{\partial(-E)} \frac{\partial W}{\partial t}=-1
$$

The transformation is now canonical with scale factor -1 . The phase area element $d \varphi d W$ is constant, and Liouville's theorem holds exactly.

In terms of the new variables, the equations of motion (4.16) and (4.12) become:

$$
\begin{gathered}
\frac{d \varphi}{d s}=\omega_{\mathrm{rf}} K W \\
\frac{d W}{d s}=\Delta(s) \frac{e \hat{V}}{\omega_{\mathrm{rf}}}\left(\sin \varphi-\sin \varphi_{s}\right)
\end{gathered}
$$

In these equations, $\omega_{\mathrm{rf}}$ is a slowly varying function of $t$. In principle, we should solve Eq. (4.33) for $t$ as a function of $\varphi$ and substitute in $\omega_{\mathrm{rf}}$. Instead, we will replace $t$ by $t_{s}(s)$, the time at which the synchronous particle reaches the point $s$, so that $\omega_{\text {rf }}$ becomes a function of $s$. The same remarks apply to the voltage amplitude $\hat{V}$ whose slow time variation we also neglect. The Hamiltonian is then easily written:

$$
H_{s L}(\varphi, W ; s)=-\frac{1}{2} \omega_{\mathrm{rf} s} K W^{2}-\Delta(s) \frac{e \hat{V}_{s}}{\omega_{\mathrm{rf} s}}\left(\cos \varphi+\varphi \sin \varphi_{s}\right)
$$

where we have written $\omega_{\mathrm{rfs}}$ to indicate that this is the frequency at the synchronous time $t_{s}(s)$. Neglect of the difference between $\omega_{\mathrm{rf}}(t)$ and $\omega_{\mathrm{rf}}\left(t_{s}(s)\right)$ means that the equations of motion derived from the Hamiltonian (4.38) may not be exact, although the variables defined by Eqs. (4.33) and (4.34) are exactly canonical.

In the next section, we will derive the Hamiltonian for coupled synchrotron and betatron motion by a series of canonical transformations from the original variables. This procedure will also provide a more rigorous derivation of the results of this section. We will obtain the synchrotron variables (4.33) and (4.34), except that we will need to change the sign of $\varphi$, because the scale factor must be +1 when $x$ and $p_{x}$ are to be included in the transformation. We will take slow time variations of $\omega_{\text {rf }}$ and $\hat{V}$ into account to first order, and we will expand the Hamiltonian to fourth order in $W$.

In order to be able to study synchrotron motion near the transition energy where $K$ vanishes, it will be convenient to add to Eq. (4.38) the third-order term from Eq. (5.68):

$$
H_{s L}(\varphi, W ; s)=\frac{1}{2} \omega_{\mathrm{rf} s} K W^{2}-K_{3} W^{3}+\Delta(s) \frac{e \hat{V}_{s}}{\omega_{\mathrm{rf} s}}\left(\cos \varphi+\varphi \sin \varphi_{s}\right)
$$


where the coefficient $K_{3}$ is given by Eq. (5.70).

The corresponding difference equations are

$$
\begin{aligned}
\varphi_{j+1} & =\varphi_{j}+\left(s_{j+1}-s_{j}\right)\left(\omega_{\mathrm{rf}} K W_{j}-3 K_{3} W_{j}^{2}\right), \\
W_{j+1} & =W_{j}+\frac{e \hat{V}_{s}}{\omega_{\mathrm{rf}}}\left(\sin \varphi_{j+1}-\sin \varphi_{s}\right) .
\end{aligned}
$$

The smooth Hamiltonians corresponding to Eqs. (4.26) and (4.31) are:

$$
\begin{aligned}
H_{s L S}(\varphi, W ; s)= & \frac{1}{2} \omega_{\mathrm{rfs}} K W^{2}-\left\langle K_{3}\right\rangle_{\mathrm{av}} W^{3}+\frac{e \hat{V}_{s}}{2 \pi R \omega_{\mathrm{rf} s}}\left(\cos \varphi+\varphi \sin \varphi_{s}\right) \\
& -\left(\frac{1}{\gamma^{2}}-f\right) \frac{e g_{0}}{4 \pi \epsilon_{0} \beta c} \lambda(\varphi), \\
H_{t L S}(\varphi, W ; s)= & \frac{1}{2} \beta c \omega_{\mathrm{rf} s} K W^{2}-\beta c\left\langle K_{3}\right\rangle_{\mathrm{av}} W^{3}+\frac{e \hat{V}_{s}}{2 \pi h}\left(\cos \varphi+\varphi \sin \varphi_{s}\right) \\
& -\left(\frac{1}{\gamma^{2}}-f\right) \frac{e g_{0}}{4 \pi \epsilon_{0}} \lambda(\varphi),
\end{aligned}
$$

where we have set $L=2 \pi R$ because these equations apply to circular accelerators, and have used Eq. (5.18).

\section{SYNCHROBETATRON COUPLING}

In this section we will follow the method of treatment used by Suzuki [10]. The particular case we treat and the approximations we make differ from those of Suzuki.

\subsection{Coupling Hamiltonian in Original Variables}

In studying synchrobetatron coupling, we will use $s$ as the independent variable. We could also use $t$, but there are advantages in using $s$, particularly in handling the rf gaps. In order to study motion near the transition energy, we will need to keep at least third-order terms in the energy difference. In order to study synchrotron sidebands near the nonlinear betatron resonances, we need nonlinear terms in the betatron variables. We will keep up to fourth-order terms in the energy difference and the betatron variables.

Proper handling of the slowly varying rf voltage and magnetic fields is a tricky matter, particularly since they are given to us as functions of $t$, which is a dynamical variable. We want the slowly varying quantities to be functions of the independent variable $s$. We therefore expand them about the synchronous time $t_{s}$ to first order in $t-t_{s}$, that is, we will keep only terms involving the first time derivatives. Because $\frac{d p}{d t}=\frac{d E}{d s}$ [see Eq. (5.21) below], the time derivative $\dot{p_{0}}$ is of the order of (or smaller than) the synchrotron variables. We will take all time derivatives to be of the order of the betatron and synchrotron variables in determining which terms to keep in the derivation. To simplify the algebra, we will assume that all magnetic fields $(B, Q, S, O)$ have the same slow time dependence. We will take them all to be proportional to $p_{0}(t)$, which is proportional to $B(t)$ according to Eq. (1.1). There are then usually only two independent time dependences, that of $p_{0}$ and that of the rf voltage amplitude. The design radio frequency $\omega_{\mathrm{rf}}(t)$ can be expressed in terms of the synchronous momentum [Eq. (5.76)]. However, if there are rf errors, or if we manipulate $\omega_{\mathrm{rf}}(t)$ in order to move the beam around in the vacuum chamber, as in beam stacking, then Eq. (5.76) no longer holds exactly. We will therefore keep separate the time derivatives of $\omega_{\mathrm{rf}}(t)$ and $p_{0}$. For 
completeness, since we are doing all this algebra anyway, we will also include radiation loss, which may be important in electron accelerators.

To handle these matters, we will start from the exact Hamiltonian (1.11), with $y$ motion omitted, and derive the Hamiltonian for synchrobetatron coupling by a sequence of canonical transformations, without making any approximations except for the expansions to fourth order in the dynamical variables and the time derivatives of slowly varying quantities.

The Hamiltonian (1.11) with the $y$ motion omitted is

$$
H_{s}\left(\underline{x}, \underline{p_{x}}, t,-E ; s\right)=-e A_{s}-p\left(1+\frac{\underline{x}}{\rho}\right)\left[1-\frac{{\underline{p_{x}}}^{2}}{p^{2}}\right]^{1 / 2},
$$

where we use $\underline{x}$ for the horizontal distance measured from the reference orbit. We assume bends with edges perpendicular to the reference orbit $(\alpha=0)$ and expand up to fourth order in the betatron variables:

$$
\begin{aligned}
H_{s}\left(\underline{x}, \underline{p_{x}}, t,-E ; s\right)= & -p+e \Delta(s) \int_{0}^{t} V(t) d t \\
& -\frac{p-p_{0}}{\rho} \underline{x}+\frac{1}{2 p} \underline{p}_{x}^{2}+\frac{1}{2} k p_{0} \underline{x}^{2}+H_{N L B 0},
\end{aligned}
$$

where the first line contains only longitudinal variables, and the second line contains the transverse variables and $p$, which depends on E [see Eq. (3.2)]. The parameter $k$ is given by

$$
k(s)=\frac{1}{\rho^{2}}-\frac{Q}{p_{0}} .
$$

Because the field gradient $Q$ increases in proportion to $p_{0}, k(s)$ is independent of time.

Nonlinear terms in the betatron equations of motion are given by cubic and quartic terms in the Hamiltonian:

$$
H_{N L B 0}=\frac{e S}{6} \underline{x}^{3}+\frac{1}{2 p \rho} \underline{x} \underline{p}_{x}^{2}-\frac{e Q^{\prime \prime}+12 e O}{48} \underline{x}^{4}+\frac{1}{8 p^{3}}{\underline{p_{x}}}^{4}
$$

\subsection{Transformation to Synchronous Coordinates}

A particle on the reference orbit $\left(\underline{x}=0, \underline{p_{x}}=0\right)$ must have $p=p_{0}$. We define the reference energy

$$
E_{0}(t)=\left[p_{0}^{2} c^{2}+m^{2} c^{4}\right]^{1 / 2}
$$

and the reference velocity

$$
\beta_{0}(t)=\frac{p_{0} c}{E_{0}}=\left[1+\frac{m^{2} c^{2}}{p_{0}^{2}}\right]^{-1 / 2} .
$$

We define a synchronous particle that follows the reference orbit and whose time variable $t_{s}(s)$ must satisfy

$$
\frac{d t_{s}}{d s}=-\frac{\partial H_{s}}{\partial E}=\frac{\partial p}{\partial E}=\frac{E_{0}\left(t_{s}\right)}{p_{0}\left(t_{s}\right) c^{2}}=\frac{1}{\beta_{0}\left(t_{s}\right) c} .
$$

The solution of this equation allows us to write the energy of the synchronous particle as a function of $s$ :

$$
E_{s}(s)=E_{0}\left(t=t_{s}(s)\right)
$$


We will use the subscript $s$ to denote slowly varying quantities evaluated at time $t=t_{s}(s)$. The reference momentum $p_{0}$ is defined by Eq. (1.1) as a slowly varying function of $t$ through its dependence on the bend field $B(t)$. We expand it to lowest order in its time derivative:

$$
p_{0}(t)=p_{s}+\dot{p}_{s}\left(t-t_{s}\right)
$$

where $p_{s}=p_{0}\left(t=t_{s}\right)$ is the synchronous momentum, and $\dot{p}_{s}=\dot{p}_{0}\left(t=t_{s}\right)$.

We take for the voltage across the accelerating gap:

$$
V(t)=\hat{V}(t) \sin \int_{0}^{t} \omega_{r f}(t) d t
$$

where the gap voltage $\hat{V}(t)$ and the $\mathrm{rf}$ frequency $\omega_{\mathrm{rf}}(t)$ are slowly varying functions of $t$. We can evaluate the integral in the second term on the right in Eq. (5.2) by parts, keeping only lowest-order terms in the time variation of slowly varying quantities:

$$
\begin{aligned}
\int_{0}^{t} V(t) d t= & -\frac{\hat{V}}{\omega_{r f}} \cos \int_{0}^{t} \omega_{\mathrm{rf}}(t) d t+\frac{\omega_{\mathrm{rf}} \dot{\hat{V}}-\dot{\omega}_{\mathrm{rf}} \hat{V}}{\omega_{\mathrm{rf}}^{3}} \sin \int_{0}^{t} \omega_{\mathrm{rf}}(t) d t \\
= & -\frac{\hat{V}_{s}}{\omega_{\mathrm{rfs}}} \cos \int_{0}^{t} \omega_{\mathrm{rf}}(t) d t \\
& +\frac{\omega_{\mathrm{rfs}} \dot{\hat{V}}_{s}-\ddot{\omega}_{\mathrm{rfs}} \hat{V}_{s}}{\omega_{\mathrm{rf} s}^{3}}\left(\sin \int_{0}^{t} \omega_{\mathrm{rf}}(t) d t-\omega_{\mathrm{rfs}}\left(t-t_{s}\right) \cos \int_{0}^{t} \omega_{\mathrm{rf}}(t) d t\right) .
\end{aligned}
$$

The first line can be verified by direct differentiation of the right member. The second line comes from expanding the time dependence in the first term. We take for the gap function a periodic delta function:

$$
\Delta(s)=\sum_{j} \delta\left(s-s_{\text {gap }}-2 \pi j R\right)
$$

Let us now consider the Hamiltonian for pure synchrotron motion, represented by the first line of Eq. (5.2):

$$
H_{s 1}(t,-E ; s)=-p+e \Delta(s) \int_{0}^{t} V(t) d t
$$

We introduce new canonical variables

$$
\begin{aligned}
\underline{\varphi} & =-\int_{0}^{t} \omega_{\mathrm{rf}}(t) d t+\frac{h\left(s-s_{\text {gap }}\right)}{R} \\
W & =\frac{E-E_{s}}{\omega_{\mathrm{rf}}}
\end{aligned}
$$

via the generating function

$$
\begin{aligned}
S_{1}(t, W ; s) & =-W\left[\int_{0}^{t} \omega_{\mathrm{rf}}(t) d t-\frac{h\left(s-s_{\mathrm{gap}}\right)}{R}\right]-E_{s}\left(t-t_{s}\right)+\underline{x p} \underline{p_{x}} \\
E & =-\frac{\partial S_{1}}{\partial t}, \underline{\varphi}=\frac{\partial S_{1}}{\partial W}, \underline{x}=\frac{\partial S_{1}}{\partial \underline{p_{x}}}, \underline{p_{x}}=\frac{\partial S_{1}}{\partial \underline{x}}
\end{aligned}
$$

where the last term in Eq. (5.16) produces the identity transformation on the transverse variables. The second term in $\underline{\varphi}$ [Eq. (5.14)] is added to make $\underline{\varphi}$ a slowly varying function of $s$. The harmonic 
number $h$ is the number of oscillations of the $\mathrm{rf}$ voltage during one revolution of the synchronous particle so, at least approximately:

$$
\omega_{\mathrm{rf}}=h \frac{\beta_{0} c}{R} .
$$

For a particle at the gap $\left(s=s_{\text {gap }}+2 \pi j R\right)$, the second term in $\underline{\varphi}$ is $2 \pi j h$. Because the integral in Eq. (5.14) appears in the Hamiltonian as the argument of a trigonometric function that is multiplied by $\Delta(s)$, and $\Delta(s)$ is a periodic delta function, we may replace the integral by $-\underline{\varphi}$ :

$$
\Delta(s) \sin \int_{0}^{t} \omega_{\mathrm{rf}}(t) d t=-\Delta(s) \sin \underline{\varphi}, \Delta(s) \cos \int_{0}^{t} \omega_{\mathrm{rf}}(t) d t=\Delta(s) \cos \underline{\varphi} .
$$

Note that the sign of $\varphi$ here is opposite to that in the previous section, so that it decreases as the rf phase increases. This is necessary in order to keep the transformation (5.14), (5.15) canonical, including the betatron variables. We could have chosen instead to change the sign of $W$, but that seems less natural. In fact, $\underline{\varphi}$ now increases in the same direction as $s$, i.e., in the direction in which the beam is traveling [see Eq. (4.29)].

We define the (slowly varying) synchronous phase $\varphi_{s}$ by requiring that $E_{s}$ satisfy the equation of motion given by the Hamiltonian (5.13), with $\Delta(s)$ replaced by its average value, because $E_{s}$ is slowly varying:

$$
\frac{d E_{s}}{d s}=\frac{\partial H_{s 1}}{\partial t}=-\frac{e \hat{V}_{s}}{2 \pi R} \sin \varphi_{s},-\pi<\varphi_{s} \leq \pi,
$$

where we choose the value of $\varphi_{s}$ corresponding to the stable fixed point. Note that $E_{s}$ defined this way is not a solution of the exact equation of motion. We have smoothed out its small periodic $s$ dependence, so $E_{s}$ is not quite the energy of the synchronous particle. We may nevertheless use $E_{s}$ and $t_{s}$ as references from which to measure energy and time deviations in the following canonical transformation. This is a different choice from that in section 4, where we used the solution $E_{s}$ of the exact equation of motion as the reference. The two differ by a small periodic term. The choice is largely a matter of taste. The present choice has the advantage that the reference energy is slowly varying and is defined by Eq. (5.5), whereas the former choice required us to solve the equation of motion for $E_{s}(s)$. We note that

$$
\dot{p}_{s}=\frac{d E_{s}}{d s},
$$

either by direct calculation or by noting that both sides of this equation are equal to the force in the $s$ direction.

We can include radiation energy loss by adding it to the left side of Eq. (5.20):

$$
\frac{d E_{s}}{d s}+\frac{\Gamma}{2 \pi R}=-\frac{e \hat{V}_{s}}{2 \pi R} \sin \varphi_{s},-\pi<\varphi_{s} \leq \pi,
$$

where $\Gamma$ is the radiation loss per turn. Radiation effects cannot generally be included in the Hamiltonian for single particle motion, but in this case, where the radiation loss is a known quantity, we can include it as above, increasing $\sin \varphi_{s}$ to cover radiation energy loss. Equation (5.22) requires us to add to the Hamiltonian (5.13) the term

$$
H_{\mathrm{rad}}=-\frac{\Gamma}{2 \pi R}\left(t-t_{s}\right)
$$

where we have added a term independent of the variables in order to be able to use Eq. (5.27) below. 
We assume that $\omega_{\mathrm{rf}}(t)$ is chosen so that Eq. (5.14) also gives the synchronous phase defined by Eq. (5.22):

$$
\varphi_{s}=-\int_{0}^{t_{s}} \omega_{\mathrm{rf}}(t) d t+\frac{h\left(s-s_{\mathrm{gap}}\right)}{R}
$$

If we differentiate this equation with respect to $s$, we get

$$
\frac{d \varphi_{s}}{d s}=-\frac{\omega_{\mathrm{rf}}\left(t_{s}\right)}{\beta_{0}\left(t_{s}\right) c}+\frac{h}{R}
$$

Because $t_{s}$ is a function of $s$, this defines in principle $\omega_{\mathrm{rf}}$ as a function of its argument. Because $\varphi_{s}$ is slowly varying and never gets outside the range $-\pi<\varphi_{s} \leq \pi$, Eq. (5.25) shows that $\omega_{\mathrm{rf}}$ is given to a good approximation by Eq. (5.18), which holds exactly on average over a long period. We will use Eq. (5.18) for $\omega_{\mathrm{rf}}$ in evaluating coefficients like those in Eqs. (5.36)-(5.39) below. We will use the value of $\omega_{\mathrm{rf}}(t)$ given by Eq. (5.24) or (5.25) in the transformations to follow. If $\omega_{\text {rf }}(t)$ differs from this value, either because of errors in the rf hardware or because we use rf manipulations to move the beam off the reference orbit, then the difference would have to be added to the integrand in Eq. (5.10) and carried along as an extra term, though it would not be used in defining the transformations. We will not do this here, as it is not usually of interest in studying synchrobetatron coupling.

We will need the time difference $t-t_{s}$, which we get as follows:

$$
\begin{aligned}
\underline{\varphi}-\varphi_{s} & =-\int_{t_{s}}^{t} \omega_{\mathrm{rf}}(t) d t \\
& =-\int_{0}^{t-t_{s}} \omega_{\mathrm{rf}}\left(t_{s}+\tau\right) d \tau \\
& =-\left(t-t_{s}\right) \omega_{\mathrm{rf} s}-\int_{0}^{t-t_{s}} \tau \dot{\omega}_{\mathrm{rf}} d \tau \\
& =-\left(t-t_{s}\right) \omega_{\mathrm{rf} s}-\frac{\dot{\omega}_{\mathrm{rf} s}}{2}\left(t-t_{s}\right)^{2}
\end{aligned}
$$

We solve by successive approximations:

$$
t-t_{s}=-\frac{\underline{\varphi}-\varphi_{s}}{\omega_{\mathrm{rf} s}}-\frac{\dot{\omega}_{\mathrm{rf} s}\left(\underline{\varphi}-\varphi_{s}\right)^{2}}{2 \omega_{\mathrm{rf} s}^{3}}
$$

Note that the next term in Eq. (5.27) would be fifth order.

As an application, we treat the time dependence of the quadrupole, sextupole, and octupole coefficients, which change in proportion to $p_{0}(t)$. We use Eqs. (5.9) and (5.27) to get for $Q(t)$ :

$$
Q(t)=\frac{Q}{p_{0}}\left[p_{s}+\dot{p}_{s}\left(t-t_{s}\right)\right]=Q_{s}(s)\left[1-\frac{\dot{p}_{s}}{\omega_{\mathrm{rf} s} p_{s}}\left(\underline{\varphi}-\varphi_{s}\right)\right],
$$

where we use the fact that $Q(t) / p_{0}(t)$ is constant and keep only first-order terms in the time derivatives. Similar formulas could be written for the other multipole coefficients $S$ and $O$. Because the correction in square brackets in the right member of Eq. (5.28) is second order, it is only relevant for the terms quadratic in $\underline{x}$ in the Harniltonian. Hence in the nonlinear terms (order $\underline{x}^{3}$ and higher), we may simply replace $Q, S, O$ by $Q_{s}, S_{s}, O_{s}$.

We need a few formulas in preparation for carrying out the transformation (5.14), (5.15). The kinetic momentum $p$ may be expanded to fourth order in $W$ :

$$
p=p_{s}+p_{W} W+\frac{1}{2} p_{2 W} W^{2}+\frac{1}{6} p_{3 W} W^{3}-\frac{1}{24} p_{4 W} W^{4}
$$


where

$$
\begin{aligned}
p_{W} & \left.=\omega_{\mathrm{rf}} \frac{\partial p}{\partial E}\right)_{s} \\
p_{2 W} & \left.=\omega_{\mathrm{rf}}^{2} \frac{\partial^{2} p}{\partial E^{2}}\right)_{s} \\
p_{3 W} & \left.=\omega_{\mathrm{rf}}^{3} \frac{\partial^{3} p}{\partial E^{3}}\right)_{s} \\
p_{4 W} & \left.=-\omega_{\mathrm{rf}}^{4} \frac{\partial^{4} p}{\partial E^{4}}\right)_{s}
\end{aligned}
$$

Because $\omega_{\mathrm{rf}}$ is a slowly varying function of $t$, we expand it:

$$
\omega_{\mathrm{rf}}(t)=\omega_{\mathrm{rf} s}+\dot{\omega}_{\mathrm{rf} s}\left(t-t_{s}\right)=\omega_{\mathrm{rf} s}-\frac{\dot{\omega}_{\mathrm{rf} s}\left(\underline{\varphi}-\varphi_{s}\right)}{\omega_{\mathrm{rf} s}},
$$

where we have used Eq. (5.27). Because the term in $\dot{\omega}_{\text {rfs }}$ is second order, Eq. (5.29) can now be written as a function of $s$ and $W$ to fourth order:

$$
p=p_{s}+p_{1} W-\frac{1}{2} p_{2} W^{2}+\frac{1}{6} p_{3} W^{3}-\frac{1}{24} p_{4} W^{4}-\frac{\dot{\omega}_{\mathrm{rf} s}}{\omega_{\mathrm{rf} s}^{2}}\left(\underline{\varphi}-\varphi_{s}\right)\left(p_{1} W-p_{2} W^{2}\right),
$$

where

$$
\begin{aligned}
& \left.p_{1}(s)=\omega_{\mathrm{rf} s} \frac{\partial p}{\partial E}\right)_{s}=\frac{\omega_{\mathrm{rf} s} E_{s}}{p_{s} c^{2}}=\frac{\omega_{\mathrm{rfs}}}{\beta_{s} c}=\frac{h}{R} \\
& \left.p_{2}(s)=-\omega_{\mathrm{rf} s}^{2} \frac{\partial^{2} p}{\partial E^{2}}\right)_{s}=\frac{\omega_{\mathrm{rfs}}^{2} m^{2}}{p_{s}^{3}}=\frac{p_{1}^{2}}{p_{s} \gamma_{s}^{2}}=\frac{h^{2}}{R^{2} p_{s} \gamma_{s}^{2}} \\
& \left.p_{3}(s)=\omega_{\mathrm{rf} s}^{3} \frac{\partial^{3} p}{\partial E^{3}}\right)_{s}=\frac{3 m^{2} \omega_{\mathrm{rfs}}^{3} E_{s}}{p_{s}^{5} c^{2}}=\frac{3 h^{3}}{R^{3} p_{s}^{2} \gamma_{s}^{2}} \\
& \left.p_{4}(s)=-\omega_{\mathrm{rf} s}^{4} \frac{\partial^{4} p}{\partial E^{4}}\right)_{s}=\omega_{\mathrm{rf} s}^{4} \frac{15 m^{4} c^{4}+12 m^{2} c^{2} p_{s}^{2}}{p_{s}^{7} c^{4}}=\frac{h^{4}\left(3+12 \gamma_{s}^{2}\right)}{R^{4} p_{s}^{3} \gamma_{s}^{4}}
\end{aligned}
$$

We also need

$$
\frac{1}{p}=\frac{1}{p_{s}}-\frac{p_{1}}{p_{s}^{2}} W+\left(\frac{p_{2}}{2 p_{s}^{2}}+\frac{p_{1}^{2}}{p_{s}^{3}}\right) W^{2}+\cdots,
$$

where to second order we need no time-dependent corrections.

The Hamiltonian $H_{s 1}$, after transforming to the variables $\underline{\varphi}, W$ is:

$$
\begin{aligned}
H_{s 2}(\underline{\varphi}, W ; s)= & H_{s 1}+\frac{\partial S_{1}}{\partial s} \\
= & \frac{1}{2} p_{2} W^{2}-\frac{e \hat{V}_{s}}{\omega_{\mathrm{rf} s}} \Delta(s) \cos \underline{\varphi}+\frac{E_{s}^{\prime}}{\omega_{\mathrm{rf} s}}\left(\underline{\varphi}-\varphi_{s}\right)+\frac{\Gamma}{2 \pi R \omega_{\mathrm{rf} s}}\left(\underline{\varphi}-\varphi_{s}\right) \\
& +H_{N L S}+H_{S V S} \\
= & \frac{1}{2} p_{2} W^{2}-\frac{e \hat{V}_{s}}{\omega_{\mathrm{rfs}}} \Delta(s) \cos \underline{\varphi}-\frac{e \hat{V}_{s} \sin \varphi_{s}}{2 \pi R \omega_{\mathrm{rfs}}}\left(\underline{\varphi}-\varphi_{s}\right) \\
& +H_{N L S}+H_{S V S},
\end{aligned}
$$


where we have used Eq. (5.22), and

$$
\begin{aligned}
H_{N L S}= & -\frac{p_{3}}{6} W^{3}+\frac{p_{4}}{24} W^{4} \\
H_{S V S}= & -\frac{\dot{\omega}_{\mathrm{rf} s} e \hat{V}_{s} \sin \varphi_{s}}{4 \pi R \omega_{\mathrm{rf} s}^{3}}\left(\underline{\varphi}-\varphi_{s}\right)^{2}+\frac{\dot{\omega}_{\mathrm{rf} s}}{\omega_{\mathrm{rf} s}^{2}}\left(\underline{\varphi}-\varphi_{s}\right)\left(p_{1} W-p_{2} W^{2}\right) \\
& +\frac{e\left(\omega_{\mathrm{rf} s} \dot{\hat{V}}-\dot{\omega}_{\mathrm{rf} s} \hat{V}\right)}{\omega_{\mathrm{rf} s}^{3}} \Delta(s)\left[\sin \underline{\varphi}+\left(\underline{\varphi}-\varphi_{s}\right) \cos \underline{\varphi}+\frac{\dot{\omega}_{\mathrm{rf} s}}{\omega_{\mathrm{rf} s}^{2}}\left(\underline{\varphi}-\varphi_{s}\right)^{2} \cos \underline{\varphi}\right]
\end{aligned}
$$

contain the nonlinear terms and the low-order time dependence of the slowly varying quantities. Terms that do not contain the canonical variables may be omitted. Note that the linear terms in $W$ drop out of the Hamiltonian $H_{s 2}$, as do the linear terms in $\varphi-\varphi_{s}$ if we replace $\Delta(s)$ by its average value, because $E_{s}, \varphi_{s}$ satisfy the corresponding equations of motion. Note also that the radiation coefficient $\Gamma$ drops out of the Hamiltonian; it appears only in the definition of $\sin \varphi_{s}$.

We replace the first two terms of the Hamiltonian (5.2) by the right member of Eq. (5.41) and make the substitutions (5.14), (5.15) in the remaining terms to obtain

$$
\begin{aligned}
H_{s 3}\left(\underline{x}, \underline{p_{x}}, \underline{\varphi}, W ; s\right)= & \frac{1}{2} p_{2} W^{2}-\frac{e \hat{V}_{s}}{\omega_{\mathrm{rf}} s} \Delta(s) \cos \underline{\varphi}-\frac{e \hat{V}_{s}}{2 \pi R \omega_{\mathrm{rf} s}} \underline{\varphi} \sin \varphi_{s} \\
& -\frac{p_{1}}{\rho} \underline{x} W+\frac{1}{2 p_{s}} \underline{p}_{x}^{2}+\frac{1}{2} k p_{s} \underline{x}^{2}-\frac{\dot{p}_{s}}{\rho \omega_{\mathrm{rf} s}} \underline{x}\left(\underline{\varphi}-\varphi_{s}\right) \\
& +H_{N L S}+H_{S V S}+H_{N L B 1}+H_{N L C},
\end{aligned}
$$

where

$$
H_{N L B 1}=\frac{e S_{s}}{6} \underline{x}^{3}+\frac{1}{2 p_{s} \rho} \underline{x} \underline{p}_{x}^{2}-\frac{e Q_{s}^{\prime \prime}+12 e O_{s}}{48} \underline{x}^{4}+\frac{1}{8 p_{s}^{3}} \underline{p}_{x}^{4}
$$

and

$$
\begin{aligned}
H_{N L C}= & \frac{p_{2}}{2 \rho} \underline{x} W^{2}-\frac{p_{1}}{2 p_{s}^{2}} \underline{p}_{x}^{2} W-\frac{k \dot{p}_{s}}{2 \omega_{\mathrm{rfs}}} \underline{x}^{2}\left(\underline{\varphi}-\varphi_{s}\right)+\frac{\dot{\omega}_{\mathrm{rf} s} p_{1}}{\omega_{\mathrm{rf} s}^{2} \rho} \underline{x} W\left(\underline{\varphi}-\varphi_{s}\right) \\
& -\frac{p_{3}}{6 \rho} \underline{x} W^{3}+\frac{1}{2}\left(\frac{p_{1}^{2}}{p_{s}^{3}}+\frac{p_{2}}{2 p_{s}^{2}}\right) \underline{p}_{x}^{2} W^{2}-\frac{p_{1}}{2 p_{s}^{2} \rho} \underline{x} \underline{p}_{x}^{2} W
\end{aligned}
$$

contains nonlinear coupling terms. The last term in the second line of Eq. (5.44) is actually third order according to our definitions. It has been placed in that line because it represents a linear coupling between synchrotron and betatron motions. If we were to replace $\dot{p}_{s}=E_{s}^{\prime}$ by its value from Eq. (5.22) (as we did in Eqs. (5.41) and (5.43)), the radiation coefficient $\Gamma$ would appear explicitly in the Hamiltonian. We will therefore leave this term as it is, because radiation does not enter into the definition of $\dot{p}_{s}$.

\subsection{Transformation to Betation Coordinates}

If we ignore the slow dependences on $t$ and $s$, the linear betatron equations given by the Hamiltonian (5.44) are

$$
\frac{d \underline{x}}{d s}=\frac{p_{x}}{p_{s}}, \frac{d \underline{p_{x}}}{d s}=\frac{p_{1}}{\rho} W-k p_{s} \underline{x} .
$$

These equations have the periodic solution

$$
\underline{x}_{\mathrm{eq}}=\frac{p_{1}}{p_{s}} D(s) W,{\underline{p_{x}}}_{\mathrm{eq}}=p_{1} D^{\prime}(s) W
$$


for the off-momentum equilibrium orbit in linear approximation, where the dispersion function $D(s)$ is the periodic solution of

$$
\frac{d^{2} D}{d s^{2}}+k D=\frac{1}{\rho}
$$

We now transform to betatron coordinates:

$$
x=\underline{x}-\frac{p_{1} D}{p_{s}} W, p_{x}=\underline{p_{x}}-p_{1} D^{\prime} W,
$$

via the generating function

$$
S_{2}\left(\underline{x}, p_{x}, \underline{\varphi}, W ; s\right)=p_{x} \underline{x}-\frac{p_{1} D}{p_{s}} p_{x} W+p_{1} D^{\prime} \underline{x} W+\underline{\varphi} W-\frac{p_{1}^{2} D D^{\prime}}{2 p_{s}} W^{2}
$$

which gives for the new synchrotron variables

$$
\begin{aligned}
W & =\frac{\partial S_{2}}{\partial \underline{\varphi}}=W \\
\varphi & =\frac{\partial S_{2}}{\partial W}=\underline{\varphi}-\frac{p_{1} D}{p_{s}} p_{x}+p_{1} D^{\prime} x
\end{aligned}
$$

The new Hamiltonian is

$$
\begin{aligned}
H_{S B C}\left(x, p_{x}, \varphi, W ; s\right)= & H_{s 3}+\frac{\partial S_{2}}{\partial s} \\
= & \frac{p_{1}^{2}}{2 p_{s}}\left(\frac{1}{\gamma_{s}^{2}}-\frac{D}{\rho}\right) W^{2}-\frac{e \hat{V}_{s}}{\omega_{\mathrm{rf} s}} \Delta(s) \cos \left(\varphi+\frac{p_{1} D}{p_{s}} p_{x}-p_{1} D^{\prime} x\right) \\
& -\frac{e \hat{V}_{s}}{2 \pi R \omega_{\mathrm{rf} s}}\left(\varphi+\frac{p_{1} D}{p_{s}} p_{x}-p_{1} D^{\prime} x\right) \sin \varphi_{s}+\frac{1}{2 p_{s}} p_{x}^{2}+\frac{1}{2} k p_{s} x^{2} \\
& +H_{S 3}+H_{B 3}+H_{C 3}+H_{S V 3}+H_{S 4}+H_{B 4}+H_{C 4}+H_{S V 4}
\end{aligned}
$$

where some terms that do not contain any of the variables have been dropped, and the third- and fourth-order terms, including slowly varying terms, are

$$
\begin{aligned}
H_{S 3}= & {\left[\frac{p_{1}^{3} D^{\prime 2}}{2 p_{s}^{2}}\left(\frac{D}{\rho}-1\right)+\frac{p_{1} p_{2} D}{2 p_{s} \rho}-\frac{p_{3}}{6}+\frac{e S p_{1}^{3} D^{3}}{6 p_{s}^{3}}\right] W^{3} } \\
H_{B 3}= & \frac{e S_{s}}{6} x^{3}+\frac{1}{2 p_{s} \rho} x p_{x}^{2}, \\
H_{C 3}= & \frac{e S_{s} p_{1} D}{2 p_{s}} x^{2} W+\left(\frac{p_{2}}{2 \rho}+\frac{e S_{s} p_{1}^{2} D^{2}}{2 p_{s}^{2}}+\frac{p_{1}^{2} D^{\prime 2}}{2 p_{s} \rho}\right) x W^{2}+\frac{p_{1} D^{\prime}}{p_{s} \rho} x p_{x} W \\
& +\frac{p_{1}}{2 p_{s}^{2}}\left(\frac{D}{\rho}-1\right) p_{x}^{2} W+\frac{p_{1}^{2} D^{\prime}}{p_{s}^{2}}\left(\frac{D}{\rho}-1\right) p_{x} W^{2}, \\
H_{S V 3}= & -\frac{\dot{p}_{s}}{p_{s} \omega_{\mathrm{rf} s}}\left[\frac{p_{s}}{\rho} x\left(\underline{\varphi}-\varphi_{s}\right)-\frac{p_{1}^{2} D}{p_{s}} p_{x} W+\frac{p_{1} D}{\rho} W\left(\underline{\varphi}-\varphi_{s}\right)-\frac{p_{1}^{3} D D^{\prime}}{2 p_{s}} W^{2}\right] \\
& +\frac{e\left(\omega_{\mathrm{rf} s} \dot{\hat{V}}_{-}-\dot{\omega}_{\mathrm{rf} s} \hat{V}\right)}{\omega_{\mathrm{rf} s}^{3}} \Delta(s)\left[\sin \underline{\varphi}+\left(\underline{\varphi}-\varphi_{s}\right) \cos \underline{\varphi}+\frac{\dot{\omega}_{\mathrm{rf} s} p_{1}}{\omega_{\mathrm{rf} s}^{2}}\left(\underline{\varphi}-\varphi_{s}\right) W\right. \\
& -\frac{\dot{\omega}_{\mathrm{rf} s} e \hat{V}_{s} \sin \varphi_{s}}{4 \pi R \omega_{\mathrm{rf} s}^{3}}\left(\underline{\varphi}-\varphi_{s}\right)^{2},
\end{aligned}
$$




$$
\begin{aligned}
& H_{S 4}=\left[-\frac{p_{1}^{4} D D^{\prime 2}}{2 p_{s}^{3} \rho}+\frac{p_{4}}{24}-\left(\frac{e Q_{s}^{\prime \prime}+12 e O_{s}}{48}\right) \frac{p_{1}^{4} D^{4}}{p_{s}^{4}}+\frac{p_{1}^{4} D^{\prime 4}}{8 p_{s}^{3}}-\frac{p_{1} p_{3} D}{6 p_{s} \rho}\right. \\
& \left.+\frac{p_{1}^{2} p_{2} D^{\prime 2}}{4 p_{s}^{2}}+\frac{p_{1}^{4}}{2} \frac{D^{\prime 2}}{2 p_{s}^{3}}\right] W^{4} \\
& H_{B 4}=-\frac{e Q_{s}^{\prime \prime}+12 e O_{s}}{48} x^{4}+\frac{1}{8 p_{s}^{3}} p_{x}^{4} \\
& H_{C 4}=-\left(\frac{p_{3}}{6 \rho}+\frac{p_{1}^{3} D^{\prime 2}}{2 p_{s}^{2} \rho}\right) x W^{3}-\frac{p_{1}}{2 \rho p_{s}^{2}} x p_{x}^{2} W+\frac{p_{1} D^{\prime}}{2 p_{s}^{3}} p_{x}^{3} W-\frac{p_{1}^{2} D^{\prime}}{p_{s}^{2} \rho} x p_{x} W^{2} \\
& +\left[\frac{p_{2}}{4 p_{s}^{2}}+\frac{3 p_{1}^{2} D^{2}}{4 p_{s}^{3}}+\frac{p_{1}^{2}}{2 p_{s}^{3}}\left(1-\frac{D}{\rho}\right)\right] p_{x}^{2} W^{2} \\
& +\left(\frac{p_{1}^{3} D^{\prime 3}}{2 p_{s}^{2}}-\frac{p_{1}^{3} D D^{\prime}}{p_{s}^{3} \rho}+\frac{p_{1}^{3} D^{\prime}}{p_{s}^{3}}+\frac{p_{1} p_{2} D^{\prime}}{2 p_{s}^{2}}\right) p_{x} W^{3} \\
& -\frac{e\left(Q_{s}^{\prime \prime}+12 O_{s}\right)}{24 p_{s}^{3}}\left(2 p_{s}^{2} p_{1} D x^{3} W+3 p_{s} p_{1}^{2} D^{2} x^{2} W^{2}+2 p_{1}^{3} D^{3} x W^{3}\right), \\
& H_{S V 4}=\frac{\dot{\omega}_{\mathrm{rf} s} e\left(\omega_{\mathrm{rfs}} \dot{\hat{V}}-\dot{\omega}_{\mathrm{rfs}} \hat{V}\right)}{2 \omega_{\mathrm{rf} s}^{5}} \Delta(s)\left(\underline{\varphi}-\varphi_{s}\right)^{2} \cos \underline{\varphi} \\
& -\frac{\dot{p}_{s} k}{2 \omega_{\mathrm{rfs}}}\left(\underline{\varphi}-\varphi_{s}\right)\left[x^{2}+\frac{2 p_{1} D}{p_{s}} x W+\frac{p_{1}^{2} D^{2}}{p_{s}^{2}} W^{2}\right] \\
& +\frac{\dot{\omega}_{\mathrm{rf} s}}{\omega_{\mathrm{rfs}}^{2}}\left(\underline{\varphi}-\varphi_{s}\right)\left[\frac{p_{1}}{\rho} x W+\left(\frac{p_{1}^{2} D}{p_{s} \rho}-p_{2}\right) W^{2}\right] \text {. }
\end{aligned}
$$

We have kept the parameters $p_{1}, p_{2}, p_{3}, p_{4}$ in the coefficients in the above equations to make it clear where they came from. For the convenience of the user, we will now rewrite them, using the definitions (5.36)-(5.39). We will also split off periodic $s$ dependences in the following way. If we average the coefficient in the first term in $H_{\mathrm{SBC}}$ over the periodic dependence on $s$ and use Eqs. (5.36)-(5.39), the result is:

$$
\frac{p_{1}^{2}}{2 p_{s}}\left(\frac{1}{\gamma_{s}^{2}}-\left\langle\frac{\dot{D}}{\rho}\right\rangle_{\mathrm{av}}\right)=\frac{p_{1}^{2}}{2 p_{s}}\left(\frac{1}{\gamma_{s}^{2}}-\frac{1}{\gamma_{t}^{2}}\right)=-\frac{\omega_{\mathrm{rfs}}^{2} \eta}{2 \beta_{s}^{3} c E_{s}}=-\frac{K \omega_{\mathrm{rf} s}}{2}
$$

where $K$ is given by Eq. (4.17), $\eta$ is given by Eq. (4.23), and

$$
\frac{1}{\gamma_{t}^{2}}=\left\langle\frac{D}{\rho}\right\rangle_{\mathrm{av}}
$$

We define the periodic part of $D / \rho$ :

$$
\left(\frac{D}{\rho}\right)_{0}=\frac{D}{\rho}-\left\langle\frac{D}{\rho}\right\rangle_{\mathrm{av}}
$$

We may split the second term in $H_{S B C}$ into two terms by writing the accelerating gap function as an average plus an oscillating part:

$$
\Delta(s)=\frac{1}{2 \pi R}+\Delta_{0}(s)
$$

where for a delta-function gap the oscillating part has the Fourier expansion:

$$
\Delta_{0}(s)=\sum_{\ell=1}^{\infty} \frac{1}{\pi R} \cos \frac{\ell\left(s-s_{\text {gap }}\right)}{R}
$$


This allows us to separate the synchrotron terms in the Hamiltonian into terms with slowly varying coefficients and terms with periodic coefficients, which are often negligible.

The final form of the Hamiltonian is:

$$
\begin{aligned}
H_{S B C}\left(x, p_{x}, \varphi, W ; s\right)= & -\frac{1}{2} \omega_{\mathrm{rf} s} K W^{2}-\frac{e \hat{V}_{s}}{2 \pi R \omega_{\mathrm{rf} s}}\left(\cos \underline{\varphi}+\underline{\varphi} \sin \varphi_{s}\right)+\frac{1}{2 p_{s}} p_{x}^{2}+\frac{1}{2} k p_{s} x^{2} \\
& -\frac{h^{2}}{2 R^{2} p_{s}}\left(\frac{D}{\rho}\right)_{0} W^{2}-\frac{e \hat{V}_{s}}{\omega_{\mathrm{rf} s}} \Delta_{0}(s) \cos \underline{\varphi} \\
& +K_{3} W^{3}+\frac{e S}{6} x^{3}+\frac{1}{2 p_{s} \rho} x p_{x}^{2}+H_{C 3}+H_{S V 3} \\
& +K_{4} W^{4}-\frac{e Q^{\prime \prime}+12 e O}{48} x^{4}+\frac{1}{8 p_{s}^{3}} p_{x}^{4}+H_{C 4}+H_{S V 4}
\end{aligned}
$$

where

$$
\begin{aligned}
\underline{\varphi}= & \varphi-\frac{h D^{\prime}}{R} x+\frac{h D}{R p_{s}} p_{x} \\
K_{3}= & \frac{h^{3} D^{\prime 2}}{2 R^{3} p_{s}^{2}}\left(\frac{D}{\rho}-1\right)+\frac{h^{3} D}{2 R^{3} p_{s}^{2} \gamma_{s}^{2} \rho}-\frac{h^{3}}{2 R^{3} p_{s}^{2} \gamma_{s}^{2}}+\frac{e S h^{3} D^{3}}{6 R^{3} p_{s}^{3}} \\
K_{4}= & \frac{h^{4}\left(3+12 \gamma_{s}^{2}\right)}{24 R^{4} p_{s}^{3} \gamma_{s}^{4}}-\frac{h^{4} D}{2 R^{4} p_{s}^{3} \gamma_{s}^{2} \rho}+\frac{h^{4}\left(1+2 \gamma_{s}^{2}\right) D^{\prime 2}}{4 R^{4} p_{s}^{3} \gamma_{s}^{2}}-\frac{h^{4} D D^{\prime 2}}{2 R^{4} p_{s}^{3} \rho}+\frac{h^{4} D^{\prime 4}}{8 R^{4} p_{s}^{3}} \\
& -\frac{e\left(Q^{\prime \prime}+12 O\right) h^{4} D^{4}}{48 R^{4} p_{s}^{4}}
\end{aligned}
$$

and the slowly varying and nonlinear coupling terms are given by:

$$
\begin{aligned}
H_{C 3}= & \frac{e h S D}{2 R p_{s}} x^{2} W+\left(\frac{h^{2}}{2 R^{2} p_{s} \rho \gamma_{s}^{2}}+\frac{e h^{2} S D^{2}}{2 R^{2} p_{s}^{2}}+\frac{h^{2} D^{\prime 2}}{2 R^{2} p_{s} \rho}\right) x W^{2}+\frac{h D^{\prime}}{R p_{s} \rho} x p_{x} W \\
& +\frac{h}{2 R p_{s}^{2}}\left(\frac{D}{\rho}-1\right) p_{x}^{2} W+\frac{h^{2} D^{\prime}}{R^{2} p_{s}^{2}}\left(\frac{D}{\rho}-1\right) p_{x} W^{2}, \\
H_{S V 3}= & -\frac{\dot{p}_{s}}{\omega_{\mathrm{rf} s}}\left[\frac{1}{\rho} x\left(\underline{\varphi}-\varphi_{s}\right)-\frac{h^{2} D}{R^{2} p_{s}^{2}} p_{x} W+\frac{h D}{R p_{s} \rho} W\left(\underline{\varphi}-\varphi_{s}\right)-\frac{h^{3} D D^{\prime}}{2 R^{3} p_{s}^{2}} W^{2}\right] \\
& +\frac{e\left(\omega_{\mathrm{rf} s} \hat{\hat{V}}-\dot{\omega}_{\mathrm{rf} s} \hat{V}\right)}{\omega_{\mathrm{rf} s}^{3}} \Delta(s)\left[\sin \underline{\varphi}+\left(\underline{\varphi}-\varphi_{s}\right) \cos \underline{\varphi}\right]+\frac{\dot{\omega}_{\mathrm{rf} s} h}{R \omega_{\mathrm{rf} s}^{2}}\left(\underline{\varphi}-\varphi_{s}\right) W \\
& -\frac{\dot{\omega}_{\mathrm{rf} s} e \hat{V}_{s} \sin \varphi_{s}}{4 \pi R \omega_{\mathrm{rf} s}^{3}}\left(\underline{\varphi}-\varphi_{s}\right)^{2}, \\
H_{C 4}= & -\left(\frac{h^{3}}{2 R^{3} p_{s}^{2} \gamma_{s}^{2} \rho}+\frac{h^{3} D^{\prime 2}}{2 R^{3} p_{s}^{2} \rho}\right) x W^{3}-\frac{h}{2 R p_{s}^{2} \rho} x p_{x}^{2} W-\frac{h^{2} D^{\prime}}{R^{2} p_{s}^{2} \rho} x p_{x} W^{2} \\
& +\frac{h D^{\prime}}{2 R p_{s}^{3}} p_{x}^{3} W+\left[\frac{h^{2}}{4 R^{2} p_{s}^{3} \gamma_{s}^{2}}+\frac{h^{2}}{2 R^{2} p_{s}^{3}}\left(1-\frac{D}{\rho}\right)+\frac{3 h^{2} D^{\prime 2}}{4 R^{2} p_{s}^{3}}\right] p_{x}^{2} W^{2} \\
& +\left(\frac{h^{3} D^{\prime 3}}{2 R^{3} p_{s}^{3}}+\frac{h^{3} D^{\prime}\left(1+2 \gamma_{s}^{2}\right)}{2 R^{3} p_{s}^{3} \gamma_{s}^{2}}-\frac{h^{3} D D^{\prime}}{R^{3} p_{s}^{3} \rho}\right) p_{x} W^{3} \\
& -\frac{e\left(Q^{\prime \prime}+12 O\right)}{24}\left(\frac{2 h D}{R p_{s}} x^{3} W+\frac{3 h^{2} D^{2}}{R^{2} p_{s}^{2}} x^{2} W^{2}+\frac{2 h^{3} D^{3}}{R^{3} p_{s}^{3}} x W^{3}\right)
\end{aligned}
$$




$$
\begin{aligned}
H_{S V 4}= & \frac{\dot{\omega}_{\mathrm{rf} s} e\left(\omega_{\mathrm{rf} s} \dot{\hat{V}}-\dot{\omega}_{\mathrm{rf} s} \hat{V}\right)}{2 \omega_{\mathrm{rf} s}^{5}} \Delta(s)\left(\underline{\varphi}-\varphi_{s}\right)^{2} \cos \underline{\varphi} \\
& +\frac{\dot{\omega}_{\mathrm{rf} s}}{\omega_{\mathrm{rf} s}^{2}}\left(\underline{\varphi}-\varphi_{s}\right)\left[\frac{h}{R \rho} x W+\left(\frac{h^{2} D}{R^{2} p_{s} \rho}-\frac{h^{2}}{R^{2} p_{s} \gamma_{s}^{2}}\right) W^{2}\right] \\
& -\frac{\dot{p}_{s} k}{2 \omega_{\mathrm{rfs}}}\left(\underline{\varphi}-\varphi_{s}\right)\left[x^{2}+\frac{2 h D}{R p_{s}} x W+\frac{h^{2} D^{2}}{R^{2} p_{s}^{2}} W^{2}\right]
\end{aligned}
$$

Note that the first line of the Hamiltonian (5.68) has the form of the uncoupled Hamiltonian for smooth synchrotron and linear betatron motion, if we replace $\varphi$ by $\varphi$. The second line contains periodic synchrotron terms with period $2 \pi R$, which are often neglected. The slight differences from Eq. (4.18) are due to differences in the definitions of $W$ and $\varphi$, mostly required in order to keep the transformations canonical. The coupling and nonlinear terms come from quantities defined in Eqs. (5.69)-(5.75).

Whew!

We can relate $\dot{\omega}_{\text {rfs }}$ to $\dot{p}_{s}$ in Eqs. (5.73) and (5.75) when Eq. (5.18) is an adequate approximation:

$$
\begin{gathered}
\omega_{\mathrm{rf}}=\frac{h p_{0} c^{2}}{R E_{0}}=\frac{h c}{R}\left(1+\frac{m^{2} c^{2}}{p_{0}^{2}}\right)^{-1 / 2} \\
\dot{\omega}_{\mathrm{rf} s}=\frac{h m^{2} c^{3} \dot{p}_{s}}{R p_{s}^{3}}\left(1+\frac{m^{2} c^{2}}{p_{s}^{2}}\right)^{-3 / 2}=\frac{h m^{2} c^{6} \dot{p}_{s}}{R E_{s}^{3}}=\frac{\omega_{\mathrm{rf} s} m^{2} c^{4} \dot{p}_{s}}{p_{s} E_{s}^{2}} .
\end{gathered}
$$

\section{References}

[1] An Introduction to the Physics of High Energy Accelerators, D.A. Edwards and M.J. Syphers, pp. 129-143. John Wiley \& Sons, New York, 1993. The same material can be found in "An Introduction to the Physics of Particle Accelerators," D.A. Edwards and M.J. Syphers, AIP Conf. Proc. 184, pp. 92-105. American Institute of Physics, 1989.

[2] Accélérateurs Circulaires de Particules, H. Bruck, Presses Universitaires de France, Paris, 1966. English translation: Los Alamos National Laboratory document LA-TR-72-10, 1972.

[3] "Stability in Dynamical Systems," E.D. Courant, R.D. Ruth, and W.T. Weng, AIP Conf. Proc. 127, [Physics of High Energy Particle Accelerators,] pp. 294-343. American Institute of Physics, 1985.

[4] "Single-Particle Dynamics in Circular Accelerators," R.D. Ruth, AIP Conf. Proc. 153, [The Physics of Particle Accelerators,] pp. 150-235. American Institute of Physics, 1987.

[5] "Introduction to Nonlinear Dynamics Arising from Magnetic Monopoles," L. Michelotti, AIP Conf. Proc. 153, [The Physics of Particle Accelerators,] pp. 236-287. American Institute of Physics, 1987.

[6] "Lectures on Nonlinear Orbit Dynamics," A.J. Dragt, AIP Conf. Proc. 87, [Fermilab Summer School, 1981,] pp. 147-313. American Institute of Physics, 1982.

[7] "Applied Hamiltonian Dynamics." K. Symon, AIP Conf. Proc. 249, [The Physics of Particle Accelerators,] pp. 277-377. American Institute of Physics, 1992. 
[8] Methods of Theoretical Physics, P.M. Morse and H. Feshbach, especially Chapter 6. McGrawHill Book Co., New York, 1953.

[9] "On Intensity Limitations Imposed by Transverse Space-Charge Effects in Circular Particle Accelerators," L.J. Laslett, Proc. Summer Study on Storage Rings, Accelerators, and Experimentation at Super-High Energies, 1963, BNL Report 7534, pp. 324-367.

[10] "Equations of motion and Hamiltonian for synchrotron oscillations and coupling," T. Suzuki, KEK Report 96-10 (1996). 